\title{
Monofunctional primary amine: A new class of organocatalyst for asymmetric Aldol reaction
}

\author{
KHIANGTE VANLALDINPUIA, PORAG BORA and GHANASHYAM BEZ* \\ Department of Chemistry, North Eastern Hill University, Shillong, Meghalaya 793 022, India \\ Email: ghanashyabez@yahoo.com
}

MS received 7 September 2016; accepted 13 January 2017

\begin{abstract}
A new class of organocatalysts involving a primary amine as the only functional group is developed for catalytic asymmetric aldol reaction of cyclohexanone/cyclopentanone with various aryl aldehydes in the presence of benzoic acid as an additive at $-10^{\circ} \mathrm{C}$. In an unexpected observation, the primary amine catalyzed reactions gave excellent yield and good to excellent stereoselectivity, while secondary amines were found to have little or no reactivity under similar reaction conditions.
\end{abstract}

Keywords. D-Fructose; primary amine; monofunctional organocatalyst; asymmetric aldol.

\section{Introduction}

Recently, chiral primary amines ${ }^{1}$ have shown tremendous synthetic potential in asymmetric functionalization of sterically hindered carbonyl compounds, which cannot be functionalized using either secondary amines or metal-based catalyst. As a result, organocatalysis using bifunctional/multifunctional primary amines derived from amino acids, ${ }^{2}$ chincona, ${ }^{3}$ and carbohydrates $^{4}$ have emerged. Interestingly, most of the asymmetric organocatalysts involving amine are bifunctional or multifunctional in nature. In contrast, only a few L-proline derived pyrrolidine based monofunctional amine organocatalysts are reported for asymmetric organocatalysis. ${ }^{5}$ Unlike the bifunctional amines where the functional groups are involved in activation of both the donor and acceptor by forming highly organized transition state that controls overall stereochemical outcome of the product, controlling stereochemical outcome employing only one functional group remains a huge challenge. More so in case of the primary amine where it reacts with carbonyl compound to form a less nucleophilic and unstable iminium ion ${ }^{6}$ that have unfavorable imine-enamine equilibrium. ${ }^{7}$ To the best of our knowledge, monofunctional primary amine catalyzed asymmetric aminocatalysis has never been reported (Figure 1).

Since its discovery back in $1872^{8}$ by CharlesAldolphe Wurtz, aldol reaction has evolved as one of the most important and powerful carbon-carbon bond forming reactions in modern organic chemistry. ${ }^{9}$

*For correspondence
Moreover, the $\beta$-hydroxy carbonyl moiety that formed as a result of aldol reaction, is found in many biologically active compounds ${ }^{10}$ such as carbohydrates, antibodies, alkaloids, and terpenes.

Although numerous methods involving metal enolates ${ }^{11}$ were developed to control the stereochemical outcome of the aldol reaction, the use of L-proline as a catalyst by List et al., ${ }^{12}$ opened up an interesting new field of oragnocatalysis. Since then, various organocatalysts bearing secondary amine, ${ }^{13}$ amides,${ }^{14}$ chiral bifunctional-thioureas, ${ }^{15}$ and diamines ${ }^{16}$ have been applied for the said synthesis. Very recently, Ricci and co-workers $^{4 a}$ used chitosan aerogel as a heterogeneous organocatalyst for asymmetric direct aldol reaction in water. Zhang et al., ${ }^{4 \mathrm{~b}}$ also reported D-glucosamine derived amino alcohols catalyzed enantioselective aldol reaction of isatins with ketones in dichloromethane. Along the similar line, Peddinti et al., ${ }^{4 \mathrm{c}}$ reported the use of glucosamine derived amino alcohol to achieve organocatalytic aldol reaction. Here, we report a highly selective asymmetric aldol reaction between aryl aldehydes and cyclohexanone/cyclopentanone catalyzed by D-fructose derived monofunctional primary amine 1a (Scheme 1).

\section{Experimental}

\subsection{General remarks}

Chemicals and reagents were purchased from commercial sources and used without further purification. IR spectra were recorded on a Perkin-Elmer Spectrum One FTIR spectrometer. ${ }^{1} \mathrm{H}$ NMR $(400 \mathrm{MHz})$ and ${ }^{13} \mathrm{C}$ NMR $(100 \mathrm{MHz})$ 


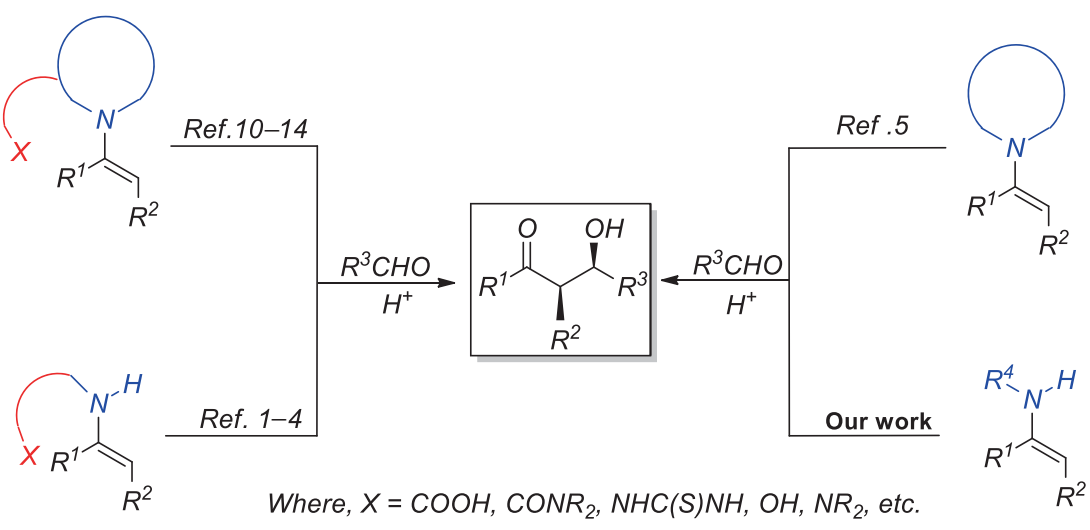

Figure 1. Schematic presentation of aminocatalysis.<smiles>[R]c1cccc(C=O)c1</smiles>

Scheme 1. Asymmetric aldol reaction catalyzed by fructose-derived amines.

spectra were obtained on a Bruker $\mathrm{AC}-400$ using $\mathrm{CDCl}_{3}$ as solvent and TMS as internal standard, unless otherwise stated. Mass spectra were obtained from Waters ZQ 4000 mass spectrometer by the ESI method, while the elemental analyses of the complexes were performed on a PerkinElmer-2400 CHN/S analyzer. Reactions were monitored by thin layer chromatography (TLC). The melting points of the compounds were recorded by open capillary method and were uncorrected. HPLC analysis was carried out on a Waters M515 equipped with Chiracel OD-H, Chiralcel AD$\mathrm{H}$, and Chiral OJ columns using $n$-hexane and 2-propanol as mobile phase at room temperature.

\subsection{Synthesis of 1,2:4,5-Di-O-isopropylidene- $\beta$ - $D$ - fructo-pyranose (3)}

To a suspension of D-fructose (36 g, $200 \mathrm{mmol})$ and 2, 2dimethoxy propane $(400 \mathrm{mmol})$ in dry acetone $(200 \mathrm{~mL})$ was added $5 \mathrm{~mol} \%$ of phosphotungstic acid and was stirred at room temperature under nitrogen atmosphere for $4 \mathrm{~h}$. After completion of the reaction, the solvent was removed under reduced pressure. Water was added to the residue and extracted with dichloromethane $(3 \times 20 \mathrm{~mL})$. The combined organic layer was dried over $\mathrm{Na}_{2} \mathrm{SO}_{4}$ and concentrated under vacuum to get the crude product, which was recrystallized by dissolving in boiling ether $(5 \mathrm{~mL} / \mathrm{g})$, cooling, and adding hexane $(5 \mathrm{~mL} / \mathrm{g})$ to give $1,2: 4,5$-di- $O$-isopropylidene- $\beta$-Dfructopyranose (37 g, 71\%). IR (KBr): 3547, 2983, 2839,
1478, 1325, 1210, 1130, 1070, $825 \mathrm{~cm}^{-1} ;{ }^{1} \mathrm{HNMR}(400$ $\left.\mathrm{MHz}, \mathrm{CDCl}_{3}\right): \delta 4.17-4.05(5 \mathrm{H}, \mathrm{m}), 3.93(1 \mathrm{H}, \mathrm{d}, J=8.8$ $\mathrm{Hz}), 3.61(1 \mathrm{H}, \mathrm{t}, J=7.6,7.6 \mathrm{~Hz}), 2.11(1 \mathrm{H}, \mathrm{d}, J=8.0$ $\mathrm{Hz}), 1.49(3 \mathrm{H}, \mathrm{s}), 1.46(3 \mathrm{H}, \mathrm{s}), 1.39(3 \mathrm{H}, \mathrm{s}), 1.32(3 \mathrm{H}, \mathrm{s})$; ${ }^{13} \mathrm{CNMR}\left(100 \mathrm{MHz}, \mathrm{CDCl}_{3}\right): \delta 112.0,109.6,104.7,28.13$, 26.62, 26.46, $26.14 \mathrm{ppm}$. ESI-MS (m/z): $283.2\left(\mathrm{M}^{+}+\mathrm{Na}\right)$; Elemental analysis for $\mathrm{C}_{12} \mathrm{H}_{20} \mathrm{O}_{6}$ : Calculated (\%) C 55.37, H 7.74; Found (\%) C 55.62, H 7.89.

\subsection{Synthesis}

2.3a 1,2:4,5-Di-O-isopropylidene-3-amino-3-deoxy- $\alpha-D-$ fructopyranose (1a) and 1,2:4,5-Di-O-isopropylidene-3amino-3-deoxy- $\beta$ - $D$-fructopyranose (2a): To a solution of $\mathrm{NiCl}_{2} .6 \mathrm{H}_{2} \mathrm{O}(9.50 \mathrm{~g}, 40 \mathrm{mmol})$ in $\mathrm{MeOH}(200 \mathrm{~mL})$ was added $\mathrm{NaBH}_{4}(1.5 \mathrm{~g}, 40 \mathrm{mmol})$ portion wise at $0^{\circ} \mathrm{C}$. The reaction mixture was stirred at $0^{\circ} \mathrm{C}$ for $30 \mathrm{~min}$ and then sugar oxime $(3,10.96 \mathrm{~g}, 40 \mathrm{mmol})$ dissolved in $\mathrm{MeOH}$ was added portion-wise along with $\mathrm{NaBH}_{4}$ (9 equiv.) for over a period of $8 \mathrm{~h}$. After completion of the reaction, the reaction mixture was run through a celite and washed with ethyl acetate. The organic portion collected was concentrated under reduced pressure to get the crude product which was purified by column chromatography using 50\% ethyl acetate in hexane as an eluent to achieve 1,2:4,5di- $O$-isopropylidene-3-amino-3-deoxy- $\beta$-D-fructopyranose (2a, $2.18 \mathrm{~g}, 21 \%$ ), followed by 1,2:4,5-di- $O$-isopropylidene3-amino-3-deoxy- $\alpha$-D-fructo-pyranose (1a, 6.42 g, 62\%). 
$2.3 \mathrm{~b} \quad 1,2: 4,5$-Di-O-isopropylidene-3-amino-3-deoxy- $\alpha-D$ fructopyranose (1a): Colourless oil, IR (KBr): 3410, 3328, 2928, 2839, 1652, 1475, 1425, 1327, 1211, 1110, 1070, $845 \mathrm{~cm}^{-1} ;{ }^{1} \mathrm{HNMR}\left(400 \mathrm{MHz}, \mathrm{CDCl}_{3}\right): \delta 4.42$ (dd, $1 \mathrm{H}, J$ $=3.2,3.6 \mathrm{~Hz}), 4.21(\mathrm{~d}, 2 \mathrm{H}, J=9.6 \mathrm{~Hz}), 3.92-3.76(\mathrm{~m}$, $4 \mathrm{H}), 3.03(\mathrm{~d}, 1 \mathrm{H}, J=2.8 \mathrm{~Hz}), 1.46(\mathrm{~s}, 3 \mathrm{H}), 1.45(\mathrm{~s}, 3 \mathrm{H})$, $1.39(\mathrm{~s}, 3 \mathrm{H}), 1.31(\mathrm{~s}, 3 \mathrm{H}) ;{ }^{13} \mathrm{CNMR}\left(125 \mathrm{MHz}, \mathrm{CDCl}_{3}\right)$ : $\delta$ 109.34, 109.10, 106.27, 73.73, 73.03, 71.88, 61.93, 52.19, 26.60, 26.12, 25.49, 24.59 ppm. ESI-MS (m/z): $260\left(\mathrm{M}^{+}\right)$; Elemental analysis for $\mathrm{C}_{12} \mathrm{H}_{21} \mathrm{NO}_{5}$ : Calculated (\%) C 55.58, H 8.16, N 5.40; Found (\%) C 55.81, H 8.29, N 5.31.

2.3c 1,2:4,5-Di-O-isopropylidene-3-amino-3-deoxy- $\beta$ - $D$ fruc-topyranose (2a): White solid, IR (KBr): 3410, 3328, 2928, 2839, 1652, 1475, 1425, 1327, 1211, 1110, 1070, $845 \mathrm{~cm}^{-1}$; ${ }^{1} \mathrm{HNMR}\left(400 \mathrm{MHz}, \mathrm{CDCl}_{3}\right): \delta 4.25(\mathrm{~d}, 1 \mathrm{H}, J=$ $8.4 \mathrm{~Hz}), 4.14-4.03$ (m, 3H), 3.95 (d, 1H, $J=8.4 \mathrm{~Hz}), 3.88$ $(\mathrm{dd}, 2 \mathrm{H}, J=1.6,1.6 \mathrm{~Hz}), 2.78(\mathrm{~d}, 1 \mathrm{H}, J=8.4 \mathrm{~Hz}), 1.51$ (s, 3H), 1.49 (s, 3H), 1.42 (s, 3H), 1.36 (s, 3H); ${ }^{13} \mathrm{CNMR}$ $\left(125 \mathrm{MHz} \mathrm{CDCl}_{3}\right): \delta$ 111.70, 109.02, 105.82, 79.39, 73.02, 72.30, 60.27, 53.87, 28.39, 26.35, 26.29, 26.14 ppm. ESIMS (m/z): $260\left(\mathrm{M}^{+}\right)$; Elemental analysis for $\mathrm{C}_{12} \mathrm{H}_{21} \mathrm{NO}_{5}$ : Calculated (\%) C 55.58, H 8.16, N 5.40; Found (\%) C 55.73, H 8.26, N 5.31.

\subsection{Synthesis of 1,2:4,5-di-O-isopropylidene-3-( $N$ - benzyl) amino-3-deoxy- $\alpha$-D-fructopyranose (1c)}

A mixture of 1,2:4,5-di- $O$-isopropylidene-3-amino-3deoxy- $\alpha$-D-fructo-pyranose $(1.3 \mathrm{~g}, 5 \mathrm{mmol})$ and benzaldehyde $(530 \mathrm{mg}, 5 \mathrm{mmol})$ was ground with a glass rod for about $10 \mathrm{~min}$ by which time, a slightly yellow solid (8) was formed. Dry dichloromethane $(20 \mathrm{~mL})$ was then added to dissolve the compound (8) followed by the addition of sodium cyanoborohydride (378 $\mathrm{mg}, 6 \mathrm{mmol})$. Upon stirring at room temperature for $6 \mathrm{~h}$, the reaction mixture was passed through celite and washed with ethyl acetate. The combined organic layers were washed with water, dried with $\mathrm{Na}_{2} \mathrm{SO}_{4}$ and concentrated under reduced pressure to give the crude product. The crude product so obtained was purified by column chromatography on silica gel using $30 \%$ ethyl acetate in hexane as an eluent to obtain 1,2:4,5:di- $O$-isopropylidene3 -( $N$-benzyl)amino-3-deoxy- $\alpha$-D-fructopyranose as a pale yellow solid (1.48 g, 88\%). IR (KBr): 3328, 3025, 2983, 2873, 1621, 1540, 1443, 1324, 1297, 1210, 1108, $847 \mathrm{~cm}^{-1}$; ${ }^{1} \mathrm{HNMR}\left(400 \mathrm{MHz}, \mathrm{CDCl}_{3}\right): \delta 7.37-7.25(\mathrm{~m}, 5 \mathrm{H}), 4.47(\mathrm{dd}$, $1 \mathrm{H}, J=2.4,2.4 \mathrm{~Hz}), 4.42(\mathrm{~d}, 1 \mathrm{H}, J=9.2 \mathrm{~Hz}), 4.20(\mathrm{~d}$, $1 \mathrm{H}, J=8.0 \mathrm{~Hz}), 4.03-3.90(\mathrm{~m}, 3 \mathrm{H}), 3.78-3.68(\mathrm{~m}, 3 \mathrm{H})$, $3.00(\mathrm{~d}, 1 \mathrm{H}, J=2.4 \mathrm{~Hz}), 1.49(\mathrm{~s}, 3 \mathrm{H}), 1.47(\mathrm{~s}, 3 \mathrm{H}), 1.31(\mathrm{~s}$, $3 \mathrm{H}), 1.25(\mathrm{~s}, 3 \mathrm{H}) ;{ }^{13} \mathrm{CNMR}\left(125 \mathrm{MHz}, \mathrm{CDCl}_{3}\right): \delta 138.98$, $128.31,128.22,127.98,127.79,127.56,110.12,109.87$, $106.71,75.28,73.02,72.89,65.16,58.56,53.34,26.54$, 26.42, 26.27, 26.14 ppm. ESI-MS (m/z): $373.1\left(\mathrm{M}^{+}+\mathrm{Na}\right)$; Elemental analysis for $\mathrm{C}_{19} \mathrm{H}_{27} \mathrm{NO}_{5}$ : Calculated (\%) C 65.31, H 7.79, N 4.01; Found (\%) C 65.79, H 7.63, N 4.23.
2.5 Synthesis of 1,2:4,5-di-O-isopropylidene-3-(N-ben$z y l)$ amino-3-deoxy- $\beta$-D-fructopyranose (2c)

Compound 2c was synthesized from 1,2:4,5-di- $O$ isopropylidene-3-amino-3-deoxy- $\alpha$-D-fructopyranose $\quad(1.3$ g, $5 \mathrm{mmol}$ ) by the procedure similar to that of 1c. Pale yellow solid (1.47 g, 87\%); IR (KBr): 3328, 3025, 2983, 2873, 1621, 1540, 1443, 1324, 1297, 1210, 1108, $847 \mathrm{~cm}^{-1}$; ${ }^{1} \mathrm{HNMR}\left(400 \mathrm{MHz}, \mathrm{CDCl}_{3}\right.$ ): $\delta$ 7.30-7.15 (m, 5H), 4.39 $(\mathrm{dd}, 1 \mathrm{H}, J=2.4,2.4 \mathrm{~Hz}), 3.40(\mathrm{~d}, 1 \mathrm{H}, J=9.2 \mathrm{~Hz}), 4.11$ $(\mathrm{d}, 1 \mathrm{H}, J=7.6 \mathrm{~Hz}), 3.94-3.82(\mathrm{~m}, 3 \mathrm{H}), 3.69-3.59(\mathrm{~m}$, $3 \mathrm{H}), 2.92(\mathrm{~d}, 1 \mathrm{H}, J=2.0 \mathrm{~Hz}), 1.41(\mathrm{~s}, 3 \mathrm{H}), 1.38(\mathrm{~s}, 3 \mathrm{H})$, $1.38(\mathrm{~s}, 3 \mathrm{H}), 1.23(\mathrm{~s}, 3 \mathrm{H}) ;{ }^{13} \mathrm{CNMR}\left(125 \mathrm{MHz}, \mathrm{CDCl}_{3}\right)$ : $\delta 139.85,128.51,128.44,128.12,127.14,126.95,109.37$, $109.03,106.20,73.47,72.31,72.06,62.33,56.77,52.08$, 26.68, 26.28, 25.73, 24.74 ppm. ESI-MS (m/z): 373.1 $\left(\mathrm{M}^{+}+\mathrm{Na}\right)$; Elemental analysis for $\mathrm{C}_{19} \mathrm{H}_{27} \mathrm{NO}_{5}$ : Calculated (\%) C 65.31, H 7.79, N 4.01; Found (\%) C 65.81, H 7.61, $\mathrm{N} 4.37$.

\subsection{Synthesis of $\mathrm{N}$-Cbz-1,2:4,5-di-O-isopropylidene- 3-amino-3-deoxy- $\alpha-D$-fructopyranose $(6)^{3}$}

To a mixture of phosphotungstic acid hydrate $(0.05 \mathrm{mmol})$ and benzylchloroformate $(1.1 \mathrm{mmol})$ was added a solution of the amine 1a $(0.260 \mathrm{~g}, 1.0 \mathrm{mmol})$ in dichloromethane $(4 \mathrm{~mL})$. After stirring for $10 \mathrm{~min}$, the reaction mixture was filtered through ordinary filter paper and the filtrate was concentrated by distillation under reduced pressure. The resulting crude product was then purified by flash chromatography using $40 \%$ ethyl acetate in hexane as eluent to get the pure product (6). White solid, $0.362 \mathrm{~g}, 92 \%$ yield. IR $\left(\mathrm{CHCl}_{3}\right): 3327,3101,2928,2875,1710,1600,1537,1456$, 1372, 1230, 1088, $967 \mathrm{~cm}^{-1} ;{ }^{1} \mathrm{HNMR}\left(400 \mathrm{MHz}, \mathrm{CDCl}_{3}\right): \delta$ 7.22-7.12 (m, 5H); 5.02-4.94 (m, 1H); 4.98 (s, 2H); 4.33 (dd, $J=5.6,4.8 \mathrm{~Hz}, 1 \mathrm{H}) ; 4.07$ (dd, $J=2.4,2.0 \mathrm{~Hz}, 1 \mathrm{H}) ; 3.97-$ $3.79(\mathrm{~m}, 4 \mathrm{H}) ; 1.32(\mathrm{~s}, 3 \mathrm{H}) ; 1.29(\mathrm{~s}, 3 \mathrm{H}) ; 1.20(\mathrm{~s}, 3 \mathrm{H}) ; 1.17(\mathrm{~s}$, $3 \mathrm{H}) ;{ }^{13} \mathrm{C} \mathrm{NMR}\left(100 \mathrm{MHz}, \mathrm{CDCl}_{3}\right): \delta 156.14,136.20,128.50$, 128.30, 128.20, 111.50, 109.20, 104.45, 73.31, 71.72, 71.70, 67.09, 61.34, 51.85, 26.29, 26.09, 25.73, 24.98 ppm. ESI-MS $(\mathrm{m} / \mathrm{z}): 416.3\left(\mathrm{M}^{+}+\mathrm{Na}\right)$; Elemental analysis for $\mathrm{C}_{20} \mathrm{H}_{27} \mathrm{NO}_{7}$ : Calculated (\%) C 61.06, H 6.92, N 3.56; Found (\%) C 61.33, H 7.21, N 3.87.

\subsection{Synthesis of 1,2:4,5-Di-O-isopropylidene-3-(N- methyl) amino-3-deoxy- $\alpha$-D-fructopyranose (1b)}

To a solution of $N$-Cbz-1,2:4,5-di- $O$-isopropylidene-3amino-3-deoxy- $\alpha$-D-fructopyranose $(0.787 \mathrm{~g}, 2 \mathrm{mmol})$ in dry DMF $(5 \mathrm{~mL})$ was added $\mathrm{NaH}(0.053 \mathrm{~g}, 2.2 \mathrm{mmol})$ at $0^{\circ} \mathrm{C}$ and stirred for $10 \mathrm{~min}$. Freshly distilled MeI (0.296 g, 2.1 mmol) was then added and the reaction was allowed to run for $2 \mathrm{~h}$. After completion of the reaction, ice-cooled water $(20 \mathrm{~mL})$ was added and the compound was extracted with ethyl acetate $(20 \mathrm{~mL} \times 3)$. The combined organic layer was 
washed with brine, dried over anhydrous $\mathrm{Na}_{2} \mathrm{SO}_{4}$ and concentrated under vacuum to get the crude product, which was purified using column chromatography to obtain the product (7) as an oily liquid in 92\% yield. IR (KBr): 3018, 2991, 2980, 2892, 1712, 1600, 1550, 1455, 1382, 1240, 1087, 968 $\mathrm{cm}^{-1} ;{ }^{1} \mathrm{HNMR}\left(400 \mathrm{MHz}, \mathrm{CDCl}_{3}\right): \delta 7.38-7.29(\mathrm{~m}, 5 \mathrm{H})$, $5.16(\mathrm{~d}, 2 \mathrm{H}, J=12.0 \mathrm{~Hz}), 4.48(\mathrm{dd}, 1 \mathrm{H}, J=1.6,1.2 \mathrm{~Hz})$, 4.33-4.29 (m, 1H), $4.20(\mathrm{~d}, 1 \mathrm{H}, J=9.2 \mathrm{~Hz}), 3.99-3.87(\mathrm{~m}$, $3 \mathrm{H}), 3.72(\mathrm{~d}, 1 \mathrm{H}, J=13.6 \mathrm{~Hz}), 3.15(\mathrm{~s}, 3 \mathrm{H}), 1.49(\mathrm{~s}, 3 \mathrm{H})$, 1.44 (s, 3H), 1.32 (s, 3H), 1.25 (s, 3H); ${ }^{13} \mathrm{CNMR}(125 \mathrm{MHz}$, $\left.\mathrm{CDCl}_{3}\right): \delta 157.54,136.57,128.83,128.45,128.21,128.08$, $110.10,109.24,105.45,75.23,73.90,71.63,67.64,63.20$, 54.91, 29.70, 26.34, 26.01, 25.86, 25.19 ppm; ESI-MS (m/z): $296.3\left(\mathrm{M}^{+}+\mathrm{Na}\right)$; Elemental analysis for $\mathrm{C}_{13} \mathrm{H}_{23} \mathrm{NO}_{5}$ : Calculated (\%) C 57.13, H 8.48, N 5.12; Found (\%) C 56.81, H $8.43, \mathrm{~N} 5.23$.

To a solution of $7(0.407 \mathrm{~g}, 1 \mathrm{mmol})$ in ethyl acetate (5 mL) was added $\mathrm{Pd} / \mathrm{C}(15 \mathrm{mg} / \mathrm{mmol})$ and the reaction mixture was stirred at room temperature under hydrogen atmosphere $(1 \mathrm{~atm})$ for $24 \mathrm{~h}$. The solution was filtered and the residue was washed thoroughly with ethyl acetate. The combined filtrate was concentrated to get the crude product which was purified by column chromatography using $30 \%$ ethyl acetate in hexane as an eluent. The pure product, 1,2:4,5-Di- $O$-isopropylidene-3-( $N$-methyl)amino3-deoxy- $\beta$-D-fructopyranose (1b) was obtained as a colourless oily liquid with $87 \%$ yield $(0.237 \mathrm{~g})$. IR (KBr): 3344 , 2989, 2893, 1640, 1541, 1487, 1345, 1321, 1248, 1180, 1087, $874 \mathrm{~cm}^{-1} ;{ }^{1} \mathrm{HNMR}$ (400 MHz, $\mathrm{CDCl}_{3}$ ): $\delta 4.55$ (dd, $1 \mathrm{H}, J=1.2,0.8 \mathrm{~Hz}), 4.36(\mathrm{~d}, 1 \mathrm{H}, J=8.8 \mathrm{~Hz}), 4.15(\mathrm{~m}, 1 \mathrm{H})$, $3.83(\mathrm{~d}, 1 \mathrm{H}, J=8.8 \mathrm{~Hz}), 3.69(\mathrm{dd}, 2 \mathrm{H}, J=1.6,1.6 \mathrm{~Hz})$, $3.55(\mathrm{~d}, 1 \mathrm{H}, J=13.2 \mathrm{~Hz}), 2.61(\mathrm{~s}, 1 \mathrm{H}), 2.42(\mathrm{~s}, 3 \mathrm{H}), 1.42(\mathrm{~s}$, $3 \mathrm{H}), 1.41(\mathrm{~s}, 3 \mathrm{H}), 1.37(\mathrm{~s}, 3 \mathrm{H}), 1.25(\mathrm{~s}, 3 \mathrm{H}) ;{ }^{13} \mathrm{CNMR}(125$ $\left.\mathrm{MHz}, \mathrm{CDCl}_{3}\right): \delta 109.60,108.97,106.94,74.65,72.29,70.82$, $63.70,62.62,44.96,26.59,26.42,26.36,25.15$ ppm. ESI-MS $(\mathrm{m} / \mathrm{z}): 296.3\left(\mathrm{M}^{+}+\mathrm{Na}\right)$; Elemental analysis for $\mathrm{C}_{13} \mathrm{H}_{23} \mathrm{NO}_{5}$ : Calculated (\%) C 57.13, H 8.48, N 5.12; Found (\%) C 56.83, H 8.36, N 5.22.

\subsection{Synthesis of $N$-Cbz-1,2:4,5-di-O-isopropylidene- 3-amino-3-deoxy- $\beta$-D-fructopyranose (8)}

The compound $\mathbf{8}$ was synthesized from the amine $\mathbf{2 a}$ following similar procedure adopted for the synthesis of the compound 6. White solid, $0.358 \mathrm{~g}, 91 \%$ yield. IR $\left(\mathrm{CHCl}_{3}\right)$ : 3328, 3100, 2928, 2876, 1712, 1601, 1537, 1456, 1372, 1230, 1088, $967 \mathrm{~cm}^{-1} ;{ }^{1} \mathrm{HNMR}\left(400 \mathrm{MHz}, \mathrm{CDCl}_{3}\right): \delta 7.28-$ $7.19(\mathrm{~m}, 5 \mathrm{H}) ; 5.08(\mathrm{~d}, J=3.2 \mathrm{~Hz}, 1 \mathrm{H}) ; 5.05$ and 5.03 (both singlet, $2 \mathrm{H}, \mathrm{C}_{6} \mathrm{H}_{5} \mathrm{CH}_{2}-$ ); $4.77(\mathrm{~m}, 1 \mathrm{H}) ; 4.08-3.76$ $(\mathrm{m}, 5 \mathrm{H}) ; 1.53(\mathrm{~s}, 3 \mathrm{H}) ; 1.40(\mathrm{~s}, 3 \mathrm{H}) ; 1.31(\mathrm{~s}, 3 \mathrm{H}) ; 1.30$ $(\mathrm{s}, 3 \mathrm{H}) ;{ }^{13} \mathrm{C} \mathrm{NMR}\left(100 \mathrm{MHz}, \mathrm{CDCl}_{3}\right): \delta 156.51,136.16$, $128.58,128.53,128.44,128.30,128.19,128.05,111.79$, 109.58, 105.10, 75.94, 72.88, 72.37, 72.11, 67.16, 60.13, 52.87, 28.04, 26.44, 26.37, 26.04 ppm. ESI-MS (m/z): 416.3 $\left(\mathrm{M}^{+}+\mathrm{Na}\right)$; Elemental analysis for $\mathrm{C}_{20} \mathrm{H}_{27} \mathrm{NO}_{7}$ : Calculated (\%) C 61.06, H 6.92, N 3.56; Found (\%) C 61.39, H 7.25, N 3.84 .
2.9 Synthesis of 1,2:4,5-di-O-isopropylidene-3-(Nmethyl) amino-3-deoxy- $\beta$-D-fructopyranose $(2 \boldsymbol{b})$

The compound (2b) was synthesized from $N$-Cbz-1,2:4,5-di$O$-isopropylidene-3-amino-3-deoxy- $\beta$-D-fructopyranose (8) via the compound (9) following similar procedure adopted for the synthesis of the compound (1b).

Compound (9): Colourless gum in 95\% yield. IR (KBr): 3025, 2989, 2982, 2876, 1715, 1602, 1537, 1455, 1382, 1235, 1088, $968 \mathrm{~cm}^{-1} ;{ }^{1} \mathrm{HNMR}\left(400 \mathrm{MHz}, \mathrm{CDCl}_{3}\right): \delta 7.36-$ $7.29(\mathrm{~m}, 5 \mathrm{H}), 5.15(\mathrm{~d}, 2 \mathrm{H}, J=2.0 \mathrm{~Hz}), 4.53(\mathrm{~d}, 1 \mathrm{H}, J=$ $10.0 \mathrm{~Hz}), 4.48-4.44$ (m, 1H), 4.30-4.22 (m, 2H), 4.11-3.87 $(\mathrm{m}, 3 \mathrm{H}), 3.02(\mathrm{~d}, 3 \mathrm{H}, J=2.8 \mathrm{~Hz}), 1.56(\mathrm{~s}, 3 \mathrm{H}), 1.46(\mathrm{~s}$ $3 \mathrm{H}), 1.39$ (s, 3H), 1.33 (s, 3H); ${ }^{13} \mathrm{CNMR}\left(125 \mathrm{MHz}, \mathrm{CDCl}_{3}\right)$ : $\delta$ 156.84, 136.08, 128.54, 128.46, 128.32, 127.95, 127.62, $112.09,109.63,105.46,73.28,72.22,71.11,67.51,69.82$, 56.14, 30.21, 28.02, 26.53, 26.37, 26.26 ppm; ESI-MS (m/z): $429.3\left(\mathrm{M}^{+}+\mathrm{Na}\right)$; Elemental analysis for $\mathrm{C}_{21} \mathrm{H}_{29} \mathrm{NO}_{7}$ : Calculated C (\%) 61.90, H 7.17, N 3.44, O 27.49; Found (\%) C 62.01, H 7.21, N 3.58, O 27.76.

Compound (2b): White solid in $90 \%$ yield $(0.245 \mathrm{~g})$. IR (KBr): 3340, 2989, 2893, 1642, 1541, 1487, 1348, 1321, 1248, 1180, 1087, $874 \mathrm{~cm}^{-1} ;{ }^{1} \mathrm{HNMR}$ (400 MHz, $\left.\mathrm{CDCl}_{3}\right)$ : $\delta$ 4.61-4.57 (m, 1H), $4.36(\mathrm{~d}, 1 \mathrm{H}, J=8.0 \mathrm{~Hz}), 4.19(\mathrm{~m}$, $1 \mathrm{H}), 4.08-3.99$ (m, 3H), $3.90(\mathrm{~d}, 1 \mathrm{H}, J=8.0 \mathrm{~Hz}), 2.72(\mathrm{~d}$, $1 \mathrm{H}, J=9.2 \mathrm{~Hz}), 2.52(\mathrm{~s}, 3 \mathrm{H}), 1.55(\mathrm{~s}, 3 \mathrm{H}), 1.47(\mathrm{~s}, 3 \mathrm{H})$, 1.42 (s, 3H), 1.37 (s, 3H); ${ }^{13} \mathrm{CNMR}\left(125 \mathrm{MHz}, \mathrm{CDCl}_{3}\right): \delta$ 111.82, 108.88, 107.04, 73.68, 72.56, 72.04, 63.46, 59.79, 43.62, 28.54, 26.58, 26.49, 26.12 ppm. ESI-MS (m/z): 296.3 $\left(\mathrm{M}^{+}+\mathrm{Na}\right)$; Elemental analysis for $\mathrm{C}_{13} \mathrm{H}_{23} \mathrm{NO}_{5}$ : Calculated (\%) C 57.13, H 8.48, N 5.12; Found (\%) C 56.79, H 8.35, N 5.24 .

\subsection{Typical procedure for aldol reaction}

D-Fructose derived amine 4 (39 $\mathrm{mg}, 0.15 \mathrm{mmol}$ ), benzoic acid (18 mg, $0.15 \mathrm{mmol}$ ) and cyclohexanone (392 mg, 4 mmol) were stirred at $-10^{\circ} \mathrm{C}$ for $30 \mathrm{~min}$. Then, the $p$ nitrobenzaldehyde (151 mg, $1 \mathrm{mmol}$ ) was added and the mixture was stirred for the specified time as shown in Table 3. After completion of the reaction, the mixture was stirred vigorously with aqueous $2.0 \mathrm{~N} \mathrm{HCl}$ solution $(10 \mathrm{~mL})$ and extracted with ethyl acetate $(10 \mathrm{~mL} \times 3)$. The organic layer was washed with water, dried $\left(\mathrm{Na}_{2} \mathrm{SO}_{4}\right)$, filtered and concentrated to get the crude product which was subjected to HPLC analysis. The product was purified through flash column chromatography on silica gel (hexane/ethyl acetate) to obtain the pure aldol product, 5a in 92\% (229 mg, $0.92 \mathrm{mmol}$ ) yield.

2.10a 2-[Hydroxy-(4-nitro-phenyl)-methyl]-cyclohexanone $(5 a)^{17}$ : Yield 92\%; Syn/anti: 3/1; IR (KBr): 3435, 3330, 3025, 2889, 2789, 1720, 1602, 1547, 1440, 1320, 1284, 1220, $1180 \mathrm{~cm}^{-1} ;{ }^{1} \mathrm{HNMR}\left(400 \mathrm{MHz}, \mathrm{CDCl}_{3}\right): \delta 8.13(\mathrm{~d}$, $J=1.2 \mathrm{~Hz}, 1 \mathrm{H}), 8.12(\mathrm{~d}, J=2.4 \mathrm{~Hz}, 1 \mathrm{H}), 7.42(\mathrm{t}, J=$ $8.8,8.0 \mathrm{~Hz}, 2 \mathrm{H}), 5.44(\mathrm{~d}, J=2.0 \mathrm{~Hz}, 1 \mathrm{H}$ for $s y n), 4.83$ (d, $J=8.4 \mathrm{~Hz}, 1 \mathrm{H}$ for anti), 2.53-1.29 (m, 9H); ${ }^{13} \mathrm{CNMR}$ 
(100 MHz, $\mathrm{CDCl}_{3}$ ): $\delta 214.77$ (anti), 214.05 (syn), 149.14, 148.53, 127.87, 126.60, 123.55, 73.97 (anti), 70.08 (syn), 57.16 (anti), 56.77 (syn), 42.66, 30.73, 27.63, 25.88, 24.74 ppm; ESI-MS (m/z): $272.9\left(\mathrm{M}^{+}+\mathrm{Na}\right)$; Elemental analysis for $\mathrm{C}_{13} \mathrm{H}_{15} \mathrm{NO}_{4}$ : Calculated (\%) C 62.64, H 6.07, N 5.62; Found (\%) C 62.71, H 6.12, N 5.68. HPLC analysis Chiralcel OD-H (Hexane/ i-PrOH = 95/5, $0.5 \mathrm{~mL} / \mathrm{min}, 254 \mathrm{~nm}$, $20^{\circ} \mathrm{C}$ ): syn product $\mathrm{t}_{R}$ (minor) $56.31 \mathrm{~min}, \mathrm{t}_{R}$ (major) 64.62 min, ee: $86 \%$, anti product $\mathrm{t}_{R}$ (minor) $71.71 \operatorname{min~}_{R}$ (major) $103.06 \mathrm{~min}, e e: 62 \%$.

$2.10 \mathrm{~b}$ 2-[Hydroxy-(3-nitro-phenyl)-methyl]-cyclohexanone, $(\mathbf{5 b})^{17}$ : Yield 94\%; Syn/anti: 1.6/1; IR (KBr): 3440, 3331, 3020, 2889, 2878, 1725, 1605, 1550, 1432, 1328, 1220, 1120 $\mathrm{cm}^{-1} ;{ }^{1} \mathrm{HNMR}\left(400 \mathrm{MHz}, \mathrm{CDCl}_{3}\right): \delta 8.17-7.49(\mathrm{~m}, 4 \mathrm{H})$, $5.48(\mathrm{~d}, J=2.0 \mathrm{~Hz}, 1 \mathrm{H}$ for $s y n), 4.88(\mathrm{~d}, J=8.4 \mathrm{~Hz}, 1 \mathrm{H}$ for anti), 2.66-1.17 (m, 9H); ${ }^{13} \mathrm{CNMR}\left(100 \mathrm{MHz}, \mathrm{CDCl}_{3}\right)$ : $\delta$ 214.79 (anti), 214.03 (syn), 148.17, 143.91, 143.25, 133.94, 129.08, 121.06, 120.82, 73.88 (anti), 69.75 (syn), 57.07 (anti), 56.6 (syn), 42.43, 27.72, 25.86, 24.68 ppm; ESI-MS $(\mathrm{m} / \mathrm{z})$ : $272.6\left(\mathrm{M}^{+}+\mathrm{Na}\right)$; Elemental analysis for $\mathrm{C}_{13} \mathrm{H}_{15} \mathrm{NO}_{4}$ : Calculated (\%) C 62.64, H 6.07, N 5.62; Found (\%) C 62.69, H 6.17, N 5.68. HPLC analysis Chiralcel OD-H (Hexane/i$\left.\mathrm{PrOH}=95 / 5,0.5 \mathrm{~mL} / \mathrm{min}, 254 \mathrm{~nm}, 20^{\circ} \mathrm{C}\right):$ syn product $\mathrm{t}_{R}$ (minor) $26.30 \mathrm{~min}, \mathrm{t}_{R}$ (major) $28.20 \mathrm{~min}$, ee: $53 \%$, anti product $\mathrm{t}_{R}$ (minor) $31.13 \min \mathrm{t}_{R}$ (major) $45.12 \mathrm{~min}$, ee: $46 \%$.

2.10c 2-[Hydroxy-(4-bromo-phenyl)-methyl]-cyclohexanone $(5 c)^{17}$ : Yield 96\%; Syn/anti: 2.6/1; IR (KBr): 3445, 3320, 3018, 2889, 2877, 1725, 1602, 1540, 1423, 1322, 1240, 1184, $1120 \mathrm{~cm}^{-1} ;{ }^{1} \mathrm{HNMR}\left(400 \mathrm{MHz}, \mathrm{CDCl}_{3}\right): \delta 7.40(\mathrm{~d}, J$ $=8.4 \mathrm{~Hz}, 2 \mathrm{H}), 7.13(\mathrm{~d}, J=8.4 \mathrm{~Hz}, 2 \mathrm{H}), 5.27(\mathrm{~d}, J=2.4$ $\mathrm{Hz}, 1 \mathrm{H}$ for $s y n), 4.68(\mathrm{~d}, J=8.4 \mathrm{~Hz}, 1 \mathrm{H}), 2.48-1.18(\mathrm{~m}$, 9H); ${ }^{13} \mathrm{CNMR}\left(100 \mathrm{MHz}, \mathrm{CDCl}_{3}\right.$ ): $\delta 215.16$ (anti), 214.43 (syn), 140.51, 140.29, 131.19, 131.06, 127.72, 127.54, 121.01, 120.64, 74.03 (anti), 70.07 (syn), 57.25 (anti), 56.93 (syn), 42.98, 42.21, 27.82, 27.10, 25.87, 24.76, 24.72 ppm; ESI-MS (m/z): $307.1\left(\mathrm{M}^{+}+\mathrm{Na}\right)$; Elemental analysis for $\mathrm{C}_{13} \mathrm{H}_{15} \mathrm{BrO}_{2}$ : Calculated (\%) C 55.14, H 5.34; Found (\%) C 55.27, H 5.43. HPLC analysis Chiralcel OD-H (Hexane/ $\left.\mathrm{i}-\mathrm{PrOH}=95 / 5,0.5 \mathrm{~mL} / \mathrm{min}, 220 \mathrm{~nm}, 20^{\circ} \mathrm{C}\right)$ : syn product $\mathrm{t}_{R}$ (minor) $24.40 \mathrm{~min}, \mathrm{t}_{R}$ (major) $25.86 \mathrm{~min}$, ee: $77 \%$, anti

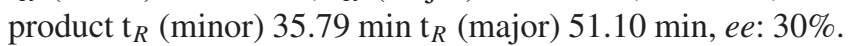

2.10d 2-[Hydroxy-(3-bromo-phenyl)-methyl]-cyclohexanone, $(5 d)^{18}$ : Yield 91\%; Syn/anti: 1.8/1; IR (KBr): 3440, 3331, 3018, 2889, 2878, 1721, 1600, 1543, 1421, 1323, 1280, 1220, 1185, $1123 \mathrm{~cm}^{-1} ;{ }^{1} \mathrm{HNMR}$ (400 MHz, $\left.\mathrm{CDCl}_{3}\right)$ : א 7.49-7.40 (m, 2H), 7.24-7.17 (m, 2H), 5.32 (d, $J=2.0 \mathrm{~Hz}$, $1 \mathrm{H}$ for $s y n), 4.75(\mathrm{~d}, J=8.4 \mathrm{~Hz}, 1 \mathrm{H}$ for anti), 2.60-1.22 (m, 9H); ${ }^{13} \mathrm{CNMR}\left(100 \mathrm{MHz}, \mathrm{CDCl}_{3}\right.$ ): $\delta 215.28$ (anti), 214.73 (syn), 143.85, 143.32, 131.84, 130.01, 129.88, 128.77, $125.75,122.56,122.45,74.29$ (anti), 70.64 (syn), 57.21, 42.62, 30.74, 27.76, 25.88, 24.67, 24.56 ppm; ESI-MS $(\mathrm{m} / \mathrm{z}): 307.3\left(\mathrm{M}^{+}+\mathrm{Na}\right)$; Elemental analysis for $\mathrm{C}_{13} \mathrm{H}_{15} \mathrm{BrO}_{2}$ : Calculated (\%) C 55.14, H 5.34; Found (\%) C 55.22, H 5.39.
HPLC analysis Chiralcel OD-H (Hexane/ i-PrOH $=95 / 5$, $0.5 \mathrm{~mL} / \mathrm{min}, 220 \mathrm{~nm}, 20^{\circ} \mathrm{C}$ ): syn product $\mathrm{t}_{R}$ (major) 15.20 min, $\mathrm{t}_{R}$ (minor) $16.24 \mathrm{~min}$, ee: $33 \%$, anti product $\mathrm{t}_{R}$ (minor) $18.58 \min _{R}$ (major) $25.51 \mathrm{~min}, e e: 44 \%$.

2.10e 2-[Hydroxy-(4-chloro-phenyl)-methyl]-cyclohexanone $(5 \boldsymbol{e})^{19}$ : Yield 90\%; Syn/anti: 2.7/1; IR (KBr): 3440, 3353, 3025, 2885, 2789, 1727, 1610, 1541, 1452, 1324, 1284, 1220, 1186, $1121 \mathrm{~cm}^{-1} ;{ }^{1} \mathrm{HNMR}\left(400 \mathrm{MHz}, \mathrm{CDCl}_{3}\right): \delta$ 7.36-7.17 (m, 4H), $5.34(\mathrm{~d}, J=2.4 \mathrm{~Hz}, 1 \mathrm{H}$ for $s y n), 4.76(\mathrm{~d}$, $J=8.0 \mathrm{~Hz}, 1 \mathrm{H}$ for anti); $2.64-1.14(\mathrm{~m}, 9 \mathrm{H}) ;{ }^{13} \mathrm{CNMR}(100$ $\mathrm{MHz} \mathrm{CDCl}_{3}$ ): $\delta 215.23$ (anti), 214.37 (syn), 139.95, 139.71, $133.49,132.86,129.98,129.78,128.41,128.20,74.07$ (anti), 70.04 (syn), 57.32 (anti), 57.05 (syn), 42.69, 42.53, 30.18, 27.86, 27.78, 25.81, 24.87, 24.73 ppm; ESI-MS $(\mathrm{m} / \mathrm{z})$ : $261.7\left(\mathrm{M}^{+}+\mathrm{Na}\right)$; Elemental analysis for $\mathrm{C}_{13} \mathrm{H}_{15} \mathrm{ClO}_{2}$ : Calculated (\%) C 65.41, H 6.33; Found (\%) C 65.23, H 5.99. HPLC analysis Chiralcel OD-H (Hexane/ i-PrOH $=90 / 10$, $0.5 \mathrm{~mL} / \mathrm{min}, 220 \mathrm{~nm}, 20^{\circ} \mathrm{C}$ ): syn product $\mathrm{t}_{R}$ (major) 12.80 min, $\mathrm{t}_{R}$ (minor) 15.32 min, ee: $81 \%$, anti product $\mathrm{t}_{R}$ (major) $20.07 \mathrm{~min}_{R}$ (minor) $22.85 \mathrm{~min}$, ee: $40 \%$.

2.10f 2-[Hydroxy-(2-chloro-phenyl)-methyl]-cyclohexanone $(5 f)^{17}$ : Yield 87\%; Syn/anti: 19/1; IR (KBr): 3441, 3325, 3018, 2889, 2877, 1721, 1602, 1541, 1423, 1320, 1248, $1185,1120 \mathrm{~cm}^{-1} ;{ }^{1} \mathrm{HNMR}\left(400 \mathrm{MHz}, \mathrm{CDCl}_{3}\right): \delta 7.53-7.23$ $(\mathrm{m}, 4 \mathrm{H}), 5.70(\mathrm{~d}, J=2.0 \mathrm{~Hz}, 1 \mathrm{H}$ for $s y n), 5.34(\mathrm{~d}, J=8.4$ $\mathrm{Hz}, 1 \mathrm{H}$ for anti), 2.83-1.32 (m, 9H); ${ }^{13} \mathrm{CNMR}(100 \mathrm{MHz}$, $\mathrm{CDCl}_{3}$ ): $\delta 215.17$ (anti), 214.58 (syn), 139.00, 138.91, $132.65,131.17,130.49,130.45,129.03,128.73,127.01$, 126.74, 70.38 (anti), 67.57 (syn), 57.84 (anti), 53.53 (syn), 42.61, 42.55, 30.31, 27.89, 27.74, 25.86, 24.89, 24.71 ppm; ESI-MS $(\mathrm{m} / \mathrm{z})$ : $261.8\left(\mathrm{M}^{+}+\mathrm{Na}\right)$; Elemental analysis for $\mathrm{C}_{13} \mathrm{H}_{15} \mathrm{ClO}_{2}$ : Calculated (\%) C 65.41, H 6.33; Found (\%) C 65.32, H 6.11. HPLC analysis Chiralcel OD-H (Hexane/ $\left.\mathrm{i}-\mathrm{PrOH}=94 / 6,0.5 \mathrm{~mL} / \mathrm{min}, 220 \mathrm{~nm}, 20^{\circ} \mathrm{C}\right)$ : syn product $\mathrm{t}_{R}$ (minor) $8.64 \mathrm{~min}, \mathrm{t}_{R}$ (major) $12.32 \mathrm{~min}$, ee: $99 \%$, anti product $\mathrm{t}_{R}$ (major) $19.50 \mathrm{~min} \mathrm{t}_{R}$ (minor) $24.43 \mathrm{~min}$, ee: $44 \%$.

$2.10 \mathrm{~g}$ 2-[Hydroxy-(2-nitro-phenyl)-methyl]-cyclohexanone $(\mathbf{5 g})^{17}$ : Yield 89\%; Syn/anti: 1/2.1; IR (KBr): 3430, 3334, 3018, 2898, 2789, 1732, 1541, 1432, 1328, 1284, 1187, $1121 \mathrm{~cm}^{-1} ;{ }^{1} \mathrm{HNMR}\left(400 \mathrm{MHz}, \mathrm{CDCl}_{3}\right): \delta 7.93-7.37(\mathrm{~m}$, $4 \mathrm{H}), 5.92(\mathrm{~d}, J=2.4 \mathrm{~Hz}, 1 \mathrm{H}$ for $s y n), 5.39(\mathrm{~d}, J=8.0$ $\mathrm{Hz}, 1 \mathrm{H}$ for anti), 2.78-1.35 (m, 9H); ${ }^{13} \mathrm{CNMR}(100 \mathrm{MHz}$, $\mathrm{CDCl}_{3}$ ): $\delta 214.56$ (anti), 213.77 (syn), 149.54, 148.65, 137.98, 134.03, 129.94, 127.67, 124.56, 69.83 (anti), 66.36 (syn), 57.21 (anti), 54.78 (syn), 42.38, 42.37, 27.32, 26.42, $24.67 \mathrm{ppm}$; ESI-MS (m/z): $272.6\left(\mathrm{M}^{+}+\mathrm{Na}\right)$; Elemental analysis for $\mathrm{C}_{13} \mathrm{H}_{15} \mathrm{NO}_{4}$ : Calculated (\%) C 62.64, H 6.07, N 5.62; Found (\%) C 62.76, H 6.17, N 5.76. HPLC analysis Chiralcel OD-H (Hexane/i-PrOH = 94/6, $0.5 \mathrm{~mL} / \mathrm{min}, 254$ $\mathrm{nm}, 20^{\circ} \mathrm{C}$ ): syn product $\mathrm{t}_{R}$ (major) $19.54 \mathrm{~min}, \mathrm{t}_{R}$ (minor) $22.77 \mathrm{~min}$, ee: $65 \%$, anti product $\mathrm{t}_{R}$ (major) $26.97 \mathrm{~min}_{R}$ (minor) $35.31 \mathrm{~min}, e e: 95 \%$. 
2.10h 2-[Hydroxy-(2-hydroxyphenyl)-methyl]-cyclohexanone (5h): Yield 87\%; Syn/anti: 2/1; IR (KBr): 3441, 3332, 3025, 2879, 1712, 1600, 1545, 1432, 1421, 1384, 1220, $1184 \mathrm{~cm}^{-1} ;{ }^{1} \mathrm{HNMR}\left(400 \mathrm{MHz}, \mathrm{CDCl}_{3}\right): \delta 7.54-6.83$ $(\mathrm{m}, 4 \mathrm{H}), 5.36(\mathrm{~d}, J=2.4 \mathrm{~Hz}, 1 \mathrm{H}$ for $s y n), 4.86(\mathrm{~d}, J=9.2$ $\mathrm{Hz}, 1 \mathrm{H}$ for anti), 2.18-0.81 (m, 9H); ${ }^{13} \mathrm{CNMR}(100 \mathrm{MHz}$, $\left.\mathrm{CDCl}_{3}\right): \delta 229.12,161.16,160.01,132.96,1321.71,130.08$, $128.42,118.79,118.47,117.34,109.94,109.89,109.44$, $109.37,67.85,63.02,61.97,51.93,26.51,26.32,26.20$, 26.07, 25.77, 25.44 ppm; ESI-MS (m/z): $243.4\left(\mathrm{M}^{+}+\mathrm{Na}\right)$; Elemental analysis for $\mathrm{C}_{13} \mathrm{H}_{16} \mathrm{O}_{3}$ : Calculated (\%) C 70.89, H 7.32, O 21.79; Found (\%) C 70.91, H 7.42, O 21.86. HPLC analysis Chiralcel OD-H (Hexane/ i-PrOH $=90 / 10$, $0.5 \mathrm{~mL} / \mathrm{min}, 220 \mathrm{~nm}, 20^{\circ} \mathrm{C}$ ): syn product $\mathrm{t}_{R}$ (major) 12.53 $\min , \mathrm{t}_{R}$ (minor) 19.83 , ee: $92 \%$; anti product $\mathrm{t}_{R}$ (minor) $23.57 \mathrm{~min} \mathrm{t}_{R}$ (major) $26.47 \mathrm{~min}, e e: 2 \%$.

2.10i 2-(Hydroxy-phenyl-methyl)-cyclohexanone $\quad(\mathbf{5 i})^{17}$ : Yield 89\%; Syn/anti: 2.7/1; IR (KBr): 3440, 3330, 3018, 2879, 1710, 1600, 1540, 1430, 1384, 1220, $1184 \mathrm{~cm}^{-1}$; ${ }^{1} \mathrm{HNMR}\left(400 \mathrm{MHz}, \mathrm{CDCl}_{3}\right.$ ): $\delta$ 7.29-7.19 (m, 4H), 5.31 (d, $J$ $=2.4 \mathrm{~Hz}, 1 \mathrm{H}$ for syn), $4.71(\mathrm{~d}, J=8.8 \mathrm{~Hz}, 1 \mathrm{H}$ for anti), 3.90 (s, 1H), 2.55-1.18 (m, 9H); ${ }^{13} \mathrm{CNMR}\left(100 \mathrm{MHz} \mathrm{CDCl}_{3}\right): \delta$ 215.55 (anti), 214.84 (syn), 140.95, 139.72, 127.89, 126.79, 126.23, 126.13, 74.74 (anti), 70.67 (syn), 57.41 (anti), 57.14 (syn), 42.62, 30.88, 27.85, 27.67, 25.88, 24.71, 24.63 ppm; ESI-MS (m/z): $227.4\left(\mathrm{M}^{+}+\mathrm{Na}\right)$; Elemental analysis for $\mathrm{C}_{13} \mathrm{H}_{16} \mathrm{O}_{2}$ : Calculated (\%) C 76.44, $\mathrm{H}$ 7.90; Found (\%) C 76.39, H 7.82. HPLC analysis Chiralcel OD-H (Hexane/ $\left.\mathrm{i}-\mathrm{PrOH}=90 / 10,0.5 \mathrm{~mL} / \mathrm{min}, 220 \mathrm{~nm}, 20^{\circ} \mathrm{C}\right)$ : syn product $\mathrm{t}_{R}$ (major) 15.12 min, $\mathrm{t}_{R}$ (minor) 16.29 , ee: $56 \%$; anti product $\mathrm{t}_{R}$ (minor) $18.82 \mathrm{~min}, \mathrm{t}_{R}$ (major) $30.07 \mathrm{~min}$, ee: $34 \%$.

\section{$2.10 \mathrm{j}$ 2-[Hydroxy-(pyridin-2-yl)-methyl]-cyclohexanone}

(5j): Yield 88\%; Syn/anti: 6/1; IR (KBr): 3450, 3343, 3100, 2898, 2789, 1724, 1600, 1537, 1474, 1398, 1321, 1286, 1224, 1189, $1121 \mathrm{~cm}^{-1}$; ${ }^{1} \mathrm{HNMR}$ (400 MHz, $\mathrm{CDCl}_{3}$ ): $\delta$ 8.21-8.08 (m, 1H), $7.67(\mathrm{~d}, J=7.6 \mathrm{~Hz}, 1 \mathrm{H}), 7.55-7.50$ (m, 2H), $7.26(\mathrm{~s}, 1 \mathrm{H}), 5.48(\mathrm{~d}, J=2.0 \mathrm{~Hz}, 1 \mathrm{H}$ for $s y n), 4.89$ (d, $J=8.4 \mathrm{~Hz}, 1 \mathrm{H}$ for anti), $3.19(\mathrm{~s}, 1 \mathrm{H}), 2.65-1.52(\mathrm{~m}, 9 \mathrm{H})$; ${ }^{13} \mathrm{CNMR}\left(100 \mathrm{MHz}, \mathrm{CDCl}_{3}\right.$ ): $\delta 214.76$ (anti), 214.21 (syn), $143.74,143.21,133.19,131.94,129.31,129.15,122.89$, 122.10, 122.02, 120.86, 74.06 (anti), 69.90 (syn), 57.13, $56.75,42.67,42.62,30.74,27.88,27.62,25.89,24.75,24.66$ ppm; ESI-MS (m/z): $229.0\left(\mathrm{M}^{+}+\mathrm{Na}\right)$; Elemental analysis for $\mathrm{C}_{12} \mathrm{H}_{15} \mathrm{NO}_{2}$ : Calculated (\%) C 70.22, H 7.37, N 6.82; Found (\%) C 70.38, H 7.58, N 7.03. HPLC analysis Chiralcel OD-H (Hexane/ i-PrOH = 47/3, $0.5 \mathrm{~mL} / \mathrm{min}, 220 \mathrm{~nm}$, $20^{\circ} \mathrm{C}$ ): syn product $\mathrm{t}_{R}$ (major) $24.51 \mathrm{~min}, \mathrm{t}_{R}$ (minor) 27.90 $\min$, ee: $32 \%$, anti product $\mathrm{t}_{R}$ (minor) $32.47 \operatorname{min~}_{R}$ (major) $37.84 \mathrm{~min}$, ee: $98 \%$.

2.10k 2-[(2-Chlorophenyl)(hydroxy)methyl]cyclopentanone, (5k): Yield 92\%; Syn/anti: 4.9/1; IR (KBr): 3330, 3025, 2887, 2784, 1742, 1600, 1472, 1380, 1322, 1286, 1224,
1182, $827 \mathrm{~cm}^{-1} ;{ }^{1} \mathrm{HNMR}\left(400 \mathrm{MHz}, \mathrm{CDCl}_{3}\right.$ ): $\delta 7.53-7.10$ $(\mathrm{m}, 4 \mathrm{H}), 5.60(\mathrm{~d}, J=2.4 \mathrm{~Hz}, 1 \mathrm{H}$ for $s y n), 5.22(\mathrm{~d}, J=$ $9.2 \mathrm{~Hz}, 1 \mathrm{H}$ for anti), 2.61-1.18 (m, 7H); ${ }^{13} \mathrm{CNMR}(100$ $\left.\mathrm{MHz} \mathrm{CDCl}_{3}\right): \delta 222.99,220.24,140.14,139.09,133.53$, $132.41,130.99,130.12,130.11,129.99,129.94,129.32$, $129.25,129.93,128.43,128.36,128.34,128.31,127.46$, $127.37,126.85,126.55,75.86,70.34,67.88,55.52,53.54$, $39.05,38.67,37.91,29.64,29.26,26.41,22.48,20.53,20.34$ ppm; ESI-MS (m/z): $247.6\left(\mathrm{M}^{+}+\mathrm{Na}\right)$; Elemental analysis for $\mathrm{C}_{12} \mathrm{H}_{13} \mathrm{ClO}_{2}$ : Calculated (\%) C 64.15, $\mathrm{H}$ 5.83, $\mathrm{Cl} 15.78$, O 14.24; Found (\%) C 63.89, H 5.91, Cl 15.65, O 14.41. HPLC analysis Chiralcel OD-H (Hexane/ i-PrOH $=47 / 3$, $0.5 \mathrm{~mL} / \mathrm{min}, 220 \mathrm{~nm}, 20^{\circ} \mathrm{C}$ ): $s y n$ product $\mathrm{t}_{R}$ (major) 20.45 $\min , \mathrm{t}_{R}$ (minor) 27.53 min, ee: $82 \%$, anti product $\mathrm{t}_{R}$ (major) $36.82 \min _{R}$ (minor) $43.17 \mathrm{~min}, e e: 78 \%$.

2.101 2-[Hydroxy(2-nitrophenyl)methyl]cyclopentanone $(5 l)^{20}$ : Yield 91\%; Syn/anti: 1.5/1; IR (KBr): 3441, 3342, 3098, 2893, 2787, 1735, 1601, 1526, 1473, 1346, 1280, 1189, $907 \mathrm{~cm}^{-1} ;{ }^{1} \mathrm{HNMR}\left(400 \mathrm{MHz}, \mathrm{CDCl}_{3}\right.$ ): $\delta 7.84-7.24$ $(\mathrm{m}, 4 \mathrm{H}), 5.92(\mathrm{~d}, J=2.0 \mathrm{~Hz}, 1 \mathrm{H}$ for $s y n), 5.44(\mathrm{~d}, J=8.4$ $\mathrm{Hz}, 1 \mathrm{H}$ for anti), 2.84-1.46 (m, 7H); ${ }^{13} \mathrm{CNMR}(100 \mathrm{MHz}$, $\left.\mathrm{CDCl}_{3}\right): \delta 221.59,219.33,148.34,147.73,137.03,136.11$, $132.59,132.31,128.81,128.73,128.23,128.04,123.85$, $123.76,69.08,65.13,56.01,51.69,39.16,38.29,26.92$, 26.83, 21.05, 20.25 ppm; ESI-MS (m/z): $258.3\left(\mathrm{M}^{+}+\mathrm{Na}\right)$; Elemental analysis for $\mathrm{C}_{12} \mathrm{H}_{13} \mathrm{NO}_{4}$ : Calculated (\%) $\mathrm{C} 61.27$, H 5.57, N 5.95, O 27.21; Found (\%) C 61.19, H 5.54, N 5.87, O 27.14. HPLC analysis Chiralcel OD-H (Hexane/i-PrOH $=47 / 3,0.5 \mathrm{~mL} / \mathrm{min}, 220 \mathrm{~nm}, 20^{\circ} \mathrm{C}$ ): syn product $\mathrm{t}_{R}$ (major) $22.21 \mathrm{~min}, \mathrm{t}_{R}$ (minor) $28.57 \mathrm{~min}$, ee: $20 \%$, anti product $\mathrm{t}_{R}$ (major) $33.80 \min _{R}$ (minor) $43.08 \mathrm{~min}$, ee: $94 \%$.

$2.10 \mathrm{~m}$ 2-[Hydroxy(p-tolyl)methyl]cyclopentanone $(\mathbf{5 m})^{21}$ : Yield 88\%; Syn/anti: 1.3/1; IR (KBr): 3340, 3100, 2989, 2898, 2789, 1745, 1601, 1535, 1482, 1397, 1324, 1280, 1222, 1189, $1120 \mathrm{~cm}^{-1} ;{ }^{1} \mathrm{HNMR}\left(400 \mathrm{MHz}, \mathrm{CDCl}_{3}\right): \delta$ $7.43-7.13(\mathrm{~m}, 4 \mathrm{H}), 5.13(\mathrm{~d}, J=2.0 \mathrm{~Hz}, 1 \mathrm{H}$ for $s y n), 4.62$ $(\mathrm{d}, J=9.2 \mathrm{~Hz}, 1 \mathrm{H}$ for $s y n), 3.00(\mathrm{~s}, 1 \mathrm{H}), 2.88(\mathrm{~m}, 1 \mathrm{H})$, 2.48-1.17 (m, 9H); ${ }^{13} \mathrm{CNMR}\left(100 \mathrm{MHz}, \mathrm{CDCl}_{3}\right): \delta 222.55$, $221.74,133.65,133.12,132.72,132.54,130.82,130.60$, $130.23,130.16,129.88,129.72,129.54,129.47,129.20$, 129.04, 128.45, 75.04, 71.53, 56.12, 55.30, 39.27, 38.77, 27.00, 26.55, 21.51, 20.17, 14.13 ppm; ESI-MS (m/z): 227.8 $\left(\mathrm{M}^{+}+\mathrm{Na}\right)$; Elemental analysis for $\mathrm{C}_{13} \mathrm{H}_{16} \mathrm{O}_{2}$ : Calculated (\%) C 76.44, H 7.90, O 15.67; Found (\%) C 76.79, H 8.11, O 15.94. HPLC analysis Chiralcel OD-H (Hexane/ i-PrOH $=47 / 3,0.5 \mathrm{~mL} / \mathrm{min}, 220 \mathrm{~nm}, 20^{\circ} \mathrm{C}$ ): syn product $\mathrm{t}_{R}$ (major) 10.54 min, $\mathrm{t}_{R}$ (minor) $13.63 \mathrm{~min}$, ee: $80 \%$, anti product $\mathrm{t}_{R}$ (major) $15.46 \operatorname{min~}_{R}$ (minor) $25.31 \mathrm{~min}$, ee: $78 \%$.

\section{Results and Discussion}

To start with, we synthesized the catalyst 1a following the Scheme 2. The acetonation of D-fructose 


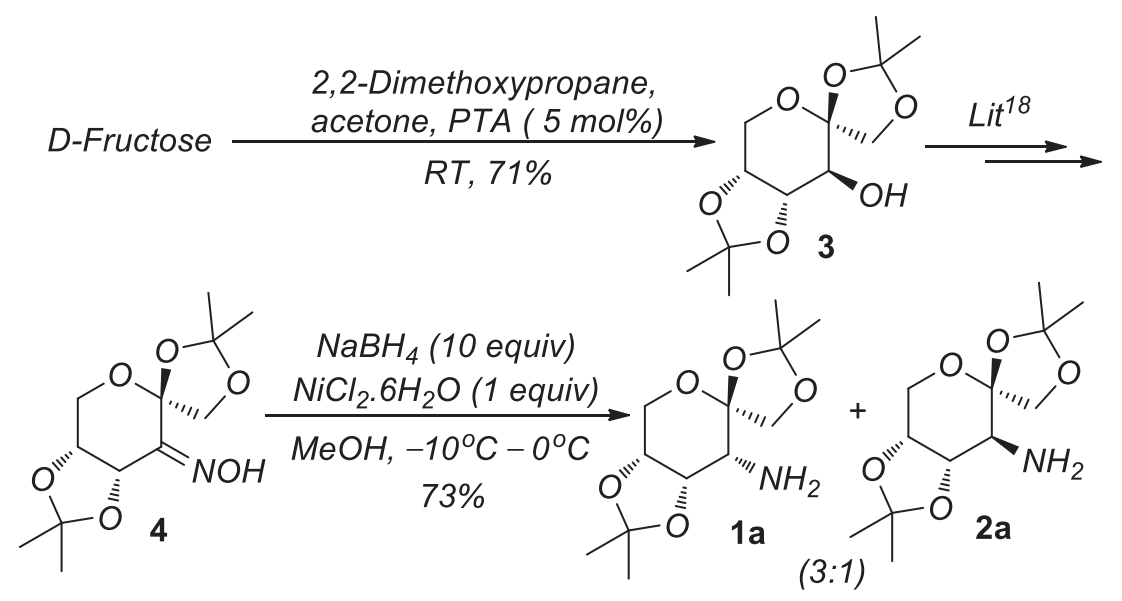

Scheme 2. Synthesis of the catalyst $\mathbf{1 a}$ and $\mathbf{2 a}$.

was accomplished by stirring D-fructose in 2,2dimethoxypropane (DMP)/acetone in the presence of a catalytic amount of phosophotungstic acid (PTA). ${ }^{22}$ The product, so obtained, was converted to oxime via Shi's ketone following literature protocol..$^{23}$ The conversion of the oxime to amine by literature procedure ${ }^{18}$ hardly gave any yield (reported yield $11 \%$ ). Therefore, we developed a new protocol to synthesize the amine by treatment of the oxime 4 with a mixture of $\mathrm{NaBH}_{4}$ (10 equiv), $\mathrm{NiCl}_{2} \cdot 6 \mathrm{H}_{2} \mathrm{O}$ (1 equiv) in methanol at -10 to $0{ }^{\circ} \mathrm{C}$ to achieve the corresponding amines in $73 \%$ yield. The yield of the amine derivative 1a was three times higher than that of its enantiomer $\mathbf{2 a}$.

In our bid to evaluate the catalytic activity of 1a, we chose the aldol reaction between p-nitrobenzaldehyde and cyclohexanone as our pilot reaction. To begin with, a mixture of the amine catalyst $1 \mathbf{a}(15 \mathrm{~mol} \%)$, benzoic acid $(15 \mathrm{~mol} \%)$ and cyclohexanone $(1 \mathrm{mmol})$ in water $(1 \mathrm{~mL})$ was stirred at room temperature for $30 \mathrm{~min}$. To this reaction mixture, p-nitrobenzaldehyde $(1 \mathrm{mmol})$ was added and the reaction was allowed to stir at room temperature for $30 \mathrm{~h}$. Ironically, the reaction resulted in very poor yield (17\%) and diastereoselectivity (syn:anti $=59: 41$ ). Assuming that the reactants might not have mixed properly in water, we screened a series of solvents, viz. ethanol, acetonitrile, methylene chloride, DMF, DMSO, and THF to observe that the reaction was insignificant in terms of yield and selectivity in most of these solvents (See Table S1 in the Supplementary Information). Interestingly, when the reaction was carried out as neat with excess amount of cyclohexanone (4 $\mathrm{mmol}$ ), the reaction was complete within $12 \mathrm{~h}$ to generate the desired product in $92 \%$ yield. HPLC analysis of the crude product indicated much improved selectivity; diastereoselectivity (syn/anti) ratio of 2.6:1 and enantiomeric excess of $21 \%$ and $15 \%$ for syn- and anti-product, respectively. Studies on the role of different acid additives

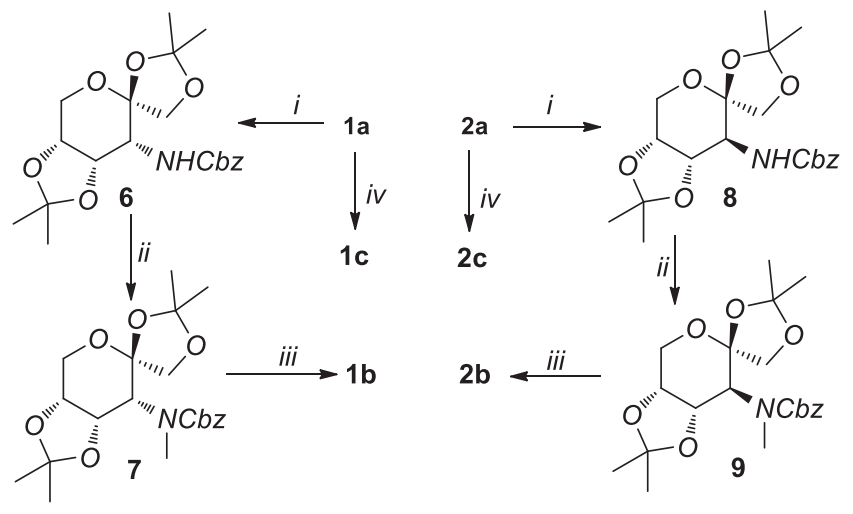

Scheme 3. Synthesis of D-fructose derived monofunctional secondary amines.

to increase the yield and selectivity of the pilot reaction confirmed that benzoic acid as the best choice (Table 1).

To improve upon the selectivity, the reaction was carried out by lowering the temperature while keeping the other parameters unchanged (entries 1-5, Table 2). The diastereomeric ratio of the syn- and anti-products and their enantioselectivities were found optimum at $-10^{\circ} \mathrm{C}$ (entry 4). Keeping in mind that the catalyst loading has a big role to play in organocatalytic reactions, ${ }^{18}$ catalytic efficiency at optimum temperature in different catalyst loading (entries 6-8) were studied to find 15 mol\% of 1a (entry 4) as the optimum loading.

Given the fact that many secondary amines have been proven as organocatalysts for various asymmetric transformations, we synthesized some secondary amines such as $\mathbf{1 b}, \mathbf{2 b}, \mathbf{1 c}$, and $\mathbf{2 c}$ (Figure 2) starting from their corresponding primary amines.

For the synthesis of monomethylated amines $\mathbf{1 b}$ and $\mathbf{2 b}$, the primary amines $\mathbf{1 a}$ and $\mathbf{2 a}$ were first protected with benzylchloroformate in the presence of a catalytic amount of phosphotungstic acid $(1 \mathrm{~mol} \%){ }^{24}$ The $\mathrm{Cbz}$ derivatives, so obtained, were methylated with MeI and 
Table 1. Effect of additives.

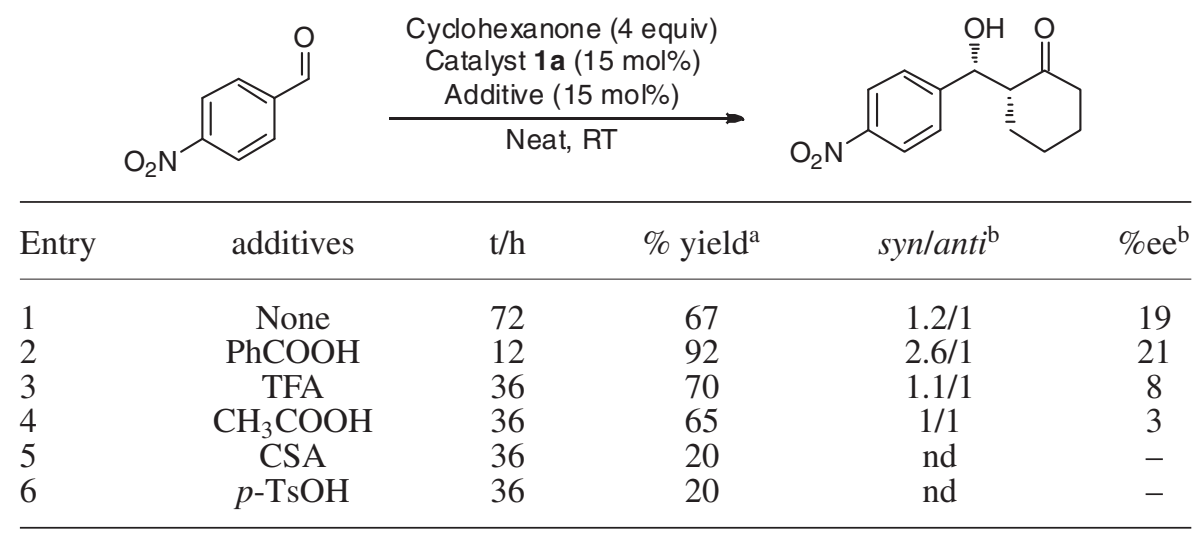

${ }^{a}$ Isolated yields.

${ }^{\mathrm{b}}$ Stereoselectivity was determined by HPLC analysis of the crude mixture using Chiralcel OD-H columns.

Table 2. Effect of temperature and catalyst loading.

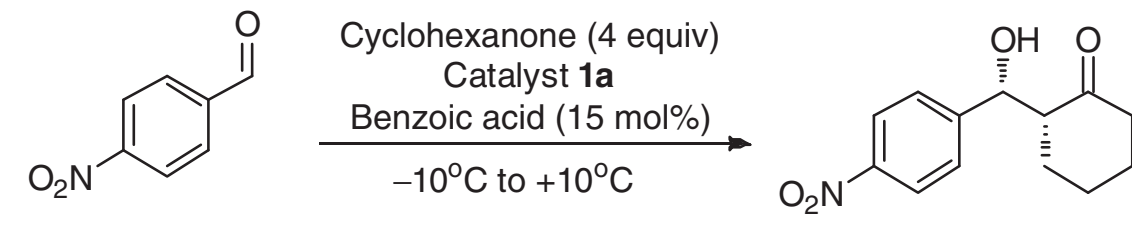

\begin{tabular}{lcccccc}
\hline Entry & $\mathbf{1 a}(\mathrm{mol} \%)$ & $\mathrm{T} /{ }^{\circ} \mathrm{C}$ & $\mathrm{t} / \mathrm{h}$ & $\%$ yield $^{\mathrm{a}}$ & syn/anti & \%ee $^{\mathrm{b}}$ \\
\hline 1 & 15 & 10 & 30 & 91 & $1.6: 1$ & 45 \\
2 & 15 & 0 & 36 & 89 & $2.6: 1$ & 70 \\
3 & 15 & -5 & 36 & 89 & $2.4: 1$ & 72 \\
4 & 15 & -10 & 36 & 92 & $3.0: 1$ & 86 \\
5 & 15 & -20 & 48 & 71 & $2.8: 1$ & 84 \\
6 & 10 & -10 & 60 & 77 & $3.0: 1$ & 86 \\
7 & 5 & -10 & 72 & 54 & $2.8: 1$ & 85 \\
8 & 20 & -10 & 36 & 94 & $2.6: 1$ & 84 \\
\hline
\end{tabular}

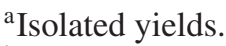

${ }^{\mathrm{b}}$ Stereoselectivity was determined by HPLC analysis of the crude mixture using Chiralcel OD-H columns.
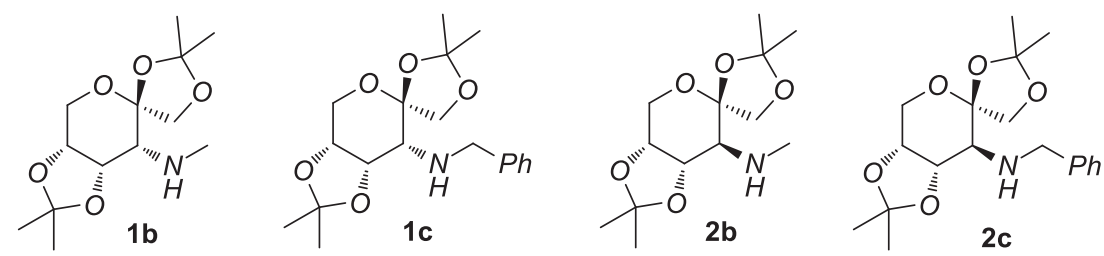

Figure 2. Fructose derived monofunctional secondary amines.

$\mathrm{NaH}$ in DMF and the resulting compounds (7 and $\mathbf{9}$, Scheme 3) were deprotected by hydrogenation on $10 \%$ $\mathrm{Pd} / \mathrm{C}$ to achieve the desired products, $\mathbf{1 b}$ and $\mathbf{2} \mathbf{b}$. The synthesis of benzylamines, $\mathbf{1 c}$ and $\mathbf{2 c}$ were accomplished by reducing a ground mixture of benzaldehyde and the primary amine in dichloromethane with sodium cyanoborohydride at room temperature.

To study the catalytic efficiency of newly synthesized monofunctional amines for asymmetric aldol reaction, the same pilot reaction was carried out under optimized reaction conditions by varying the amine catalysts. Catalyst 2a gave excellent yield (91\%) and good enantioselectivity for syn (67\%) and anti (71\%) products, but almost no diastereoselectivity was observed (Table 3). The same reaction in the presence of $N$-methyl amine derivatives $\mathbf{1 b}$ and $\mathbf{2 b}$ gave the aldol products in less than $10 \%$ yield. The pilot reaction did not proceed at all in the presence of catalysts $\mathbf{1 c}$ and 
Table 3. Screening of various amine catalysts for Aldol reaction.

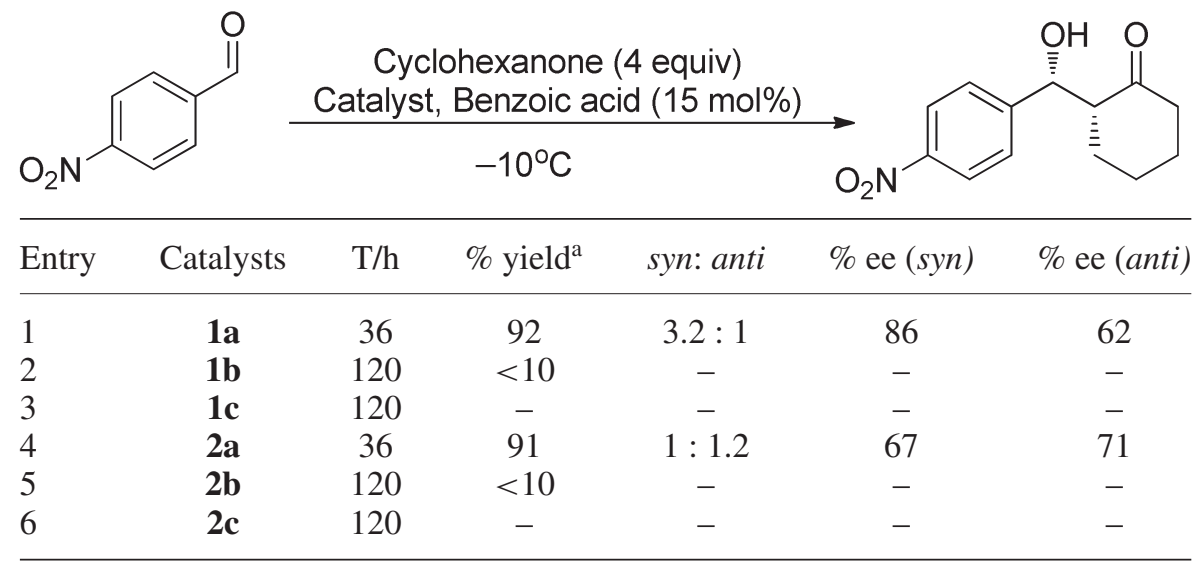

${ }^{a}$ Isolated yields.

${ }^{\mathrm{b}}$ Stereoselectivity was determined by HPLC analysis of the crude mixture using Chiralcel OD-H columns.

Table 4. Asymmetric aldol reaction catalyzed by organocatalyst 1a. ${ }^{a}$

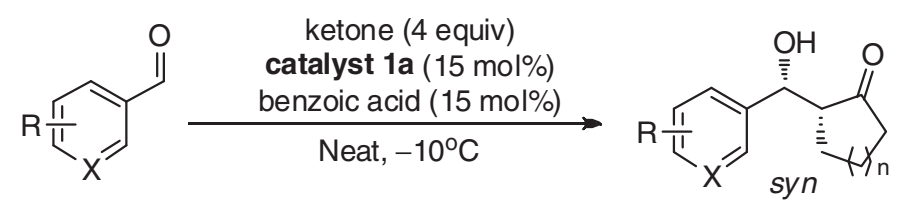

$5 a-m$

\begin{tabular}{llllllcrc}
\hline Entry & $\mathrm{R}$ & $\mathrm{X}$ & $\mathrm{n}$ & 5 & $\mathrm{t} / \mathrm{h}$ & $\%$ yield $^{\mathrm{b}}$ & syn/anti & $\%$ ee $\mathrm{c}, \mathrm{d}$ \\
\hline 1 & $p-\mathrm{NO}_{2}$ & $\mathrm{CH}$ & 2 & $\mathbf{a}$ & 36 & 92 & $3: 1$ & 86 \\
2 & $m-\mathrm{NO}_{2}$ & $\mathrm{CH}$ & 2 & $\mathbf{b}$ & 36 & 94 & $1.6: 1$ & 53 \\
3 & $p-\mathrm{Br}$ & $\mathrm{CH}$ & 2 & $\mathbf{c}$ & 36 & 96 & $2.6: 1$ & 77 \\
4 & $m-\mathrm{Br}$ & $\mathrm{CH}$ & 2 & $\mathbf{d}$ & 36 & 91 & $1.8: 1$ & 33 \\
5 & $p-\mathrm{Cl}$ & $\mathrm{CH}$ & 2 & $\mathbf{e}$ & 24 & 90 & $2.7: 1$ & 81 \\
6 & $o-\mathrm{Cl}$ & $\mathrm{CH}$ & 2 & $\mathbf{f}$ & 36 & 87 & $19: 1$ & 99 \\
7 & $o-\mathrm{NO} 2$ & $\mathrm{CH}$ & 2 & $\mathbf{g}$ & 36 & 89 & $1: 2.2$ & $95^{\mathrm{e}}$ \\
8 & $o-\mathrm{OH}$ & $\mathrm{CH}$ & 2 & $\mathbf{h}$ & 24 & 85 & $2: 1$ & 92 \\
9 & $\mathrm{H}$ & $\mathrm{CH}$ & 2 & $\mathbf{i}$ & 32 & 89 & $2.7: 1$ & 56 \\
10 & $\mathrm{H}$ & $\mathrm{N}$ & 2 & $\mathbf{j}$ & 36 & 88 & $6: 1$ & 32 \\
11 & $o-\mathrm{Cl}$ & $\mathrm{CH}$ & 1 & $\mathbf{k}$ & 24 & 92 & $4.9: 1$ & 82 \\
12 & $o-\mathrm{NO} 2$ & $\mathrm{CH}$ & 1 & $\mathbf{l}$ & 24 & 91 & $1.5: 1$ & 20 \\
13 & $p-\mathrm{Me}$ & $\mathrm{CH}$ & 1 & $\mathbf{m}$ & 24 & 88 & $1.3: 1$ & 88 \\
\hline
\end{tabular}

${ }^{a}$ Reaction conditions: aldehyde $(1 \mathrm{mmol})$, catalyst $(15 \mathrm{~mol} \%)$, benzoic acid (15 $\mathrm{mol} \%)$ and ketone (4 mmol).

${ }^{\mathrm{b}}$ Isolated yields.

${ }^{\mathrm{c}}$ Determined by HPLC analysis of the crude products using Chiralcel OD-H and AD-H columns.

$\mathrm{d} \%$ ee of the major diastereomer.

2c. Such results might be attributed to the fact that steric hindrance caused by introduction of the bulky substituents such as methyl and benzyl groups on the primary amine which might have blocked the initial formation of enamine with cyclohexanone.

After optimizing the reaction conditions such as solvent, temperature, acid additive, and catalyst loading, the substrate scope of our method was explored for asymmetric aldol reaction of cyclohexanone with a variety of aromatic aldehydes in the presence of benzoic acid (Table 4, entries 1-10). The reaction gave excellent yields in all the cases with moderate to excellent enantioselectivity for both syn- and anti- products. Especially, the aldehydes having ortho-substituents (entries 6-7, Table 4) gave very good enantioselectivities (up to $99 \%$ ), while those with meta-substituents 


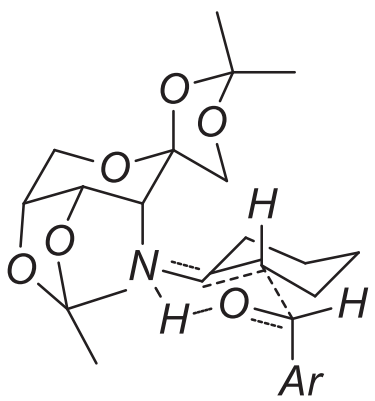

TS-1 (Favored)

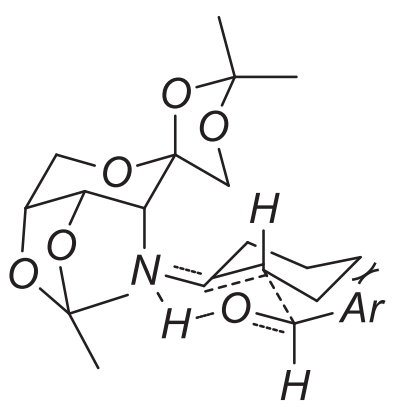

TS-2 (Less favored)
Figure 3. Plausible mechanism of syn-selectivity.

(entries 2, 4) gave poorer enantioselectivity. Interestingly, diastereoselectivity of $o$-nitrobenzaldehyde derived aldol product $(5 \mathrm{~g})$ was found to be opposite to all other aldehydes where syn- diastereomer was formed as the major product. Given the fact that cyclopentanone forms enamine faster than that of cyclohexanone, ${ }^{25}$ we screened aminocatalysis of cyclopentanone with aryl aldehydes to synthesize corresponding $\beta$-hydroxy ketones. In all the three cases, reaction time was found to be reduced substantially to give comparable diastereoselectivity with similar starting aldehydes.

The plausible mechanism to account for the stereoselectivity might be the formation of the transition states TS-1 and TS-2 wherein the catalyst 1a could catalyze the aldol reaction as shown in Figure 3. The aldehyde might be activated by hydrogen-bonding with $\mathrm{NH}$ group of cyclohexenylamine via formation of the six-membered transition states. In TS-2, the plane of aryl group might experience substantial steric hindrance due to cyclohexane ring making the transition state less favorable than TS-1. Therefore, the approach of the enamine carbon from re-face of aldehyde in TS-1 led to syn-selective aldol product.

\section{Conclusions}

In conclusion, we have established monofunctional primary amine, 1,2:4,5-di- $O$-isopropylidene-3-amino3 -deoxy- $\alpha$-D-fructopyranose (1a) as an efficient aminocatalyst for stereoselective synthesis of $\beta$ hydroxy ketones by aldol reaction. Under optimal conditions, very high diastereoselectivities (up to 95\%) and enantioselectivities (up to 99\%) were achieved. It may be noted that this is the first report on any stereoselective organocatalysis employing monofunctional primary amine, albeit monofunctional secondary amine catalysis exist in literature. Interestingly, the fructose derived secondary amines $(\mathbf{1 b}, \mathbf{1 c}, \mathbf{2 b}$, and $\mathbf{2 c})$ were found to be ineffective.

\section{Acknowledgements}

Authors acknowledge the Council of Scientific and Industrial Research (CSIR), New Delhi, India for financial support (Scheme no. 1 (1992)/05/EMR-II). The analytical services provided by Sophisticated Analytical Instrumentation Facility, North Eastern Hill University, Shillong are gratefully acknowledged.

\section{Supporting Information (SI)}

${ }^{1} \mathrm{H}$ and ${ }^{13} \mathrm{C}$ NMR spectra of new compounds and HPLC data are available free of charge at www.ias.ac.in/chemsci.

\section{References}

1. For review, see: (a) Xu L-W, Luo J and Lu Y 2009 Asymmetric catalysis with chiral primary amine-based organocatalysts Chem. Commun. 1807; (b) Peng F and Shao Z 2008 Advances in asymmetric organocatalytic reactions catalyzed by chiral primary amines $J$. Mol. Catal. A: Chem. 285 1; (c) Uehara $\mathrm{H}$ and Barbas III C F 2009 anti-Selective Asymmetric Michael Reactions of Aldehydes and Nitroolefins Catalyzed by a Primary Amine/Thiourea Angew. Chem. Int. Ed. 48 9848; (d) Bhanushali M and Zhao C-G 2011 Developing Novel Organocatalyzed Aldol Reactions for the Enantioselective Synthesis of Biologically Active Molecules Synthesis 1815; (e) Wu C, Long X, Li S and Fu X 2012 Simple and inexpensive threonine-based organocatalysts as highly active and recoverable catalysts for largescale asymmetric direct stoichiometric aldol reactions on water Tetrahedron: Asymm. 23 315; (f) Umehara A, Kanemitsu T, Nagata K and Itoh T 2012 Stereoselective Synthesis of vic-Halohydrins via L-tert-LeucineCatalyzed syn-Selective Aldol Reaction Synlett 453; (g) Jiang L and Chen Y-C 2011 Asymmetric organocatalysis mediated by primary amines derived from cinchona alkaloids: Recent advances Catal. Sci. Technol. 354; (h) Bartoli G and Melchiorre P 2008 A Novel Organocatalytic Tool for the Iminium Activation of $\alpha$, $\beta$-Unsaturated Ketones Synlett 1759

2. (a) Pizzarello S and Weber A L 2004 Prebiotic Amino Acids as Asymmetric Catalysts Science 303 1151; (b) Ramasastry S S V, Zhang H, Tanaka F and Barbas III C F 2007 Direct Catalytic Asymmetric Synthesis of anti-1,2Amino Alcohols and syn-1,2-Diols through Organocatalytic anti-Mannich and syn-Aldol Reactions J. Am. Chem. Soc. 129288

3. (a) Zhou J, Wakchaure V, Kraft P and List B 2008 Primary-Amine-Catalyzed Enantioselective Intramolecular Aldolizations Angew. Chem. Int. Ed. 47 7656; (b) Zheng B-L, Liu Q-Z, Guo C-S, Wang X-L and He L 2007 Highly enantioselective direct aldol reaction catalyzed by cinchona derived primary amines $\mathrm{Org}$. Biomol. Chem. 5 2913; (c) Li P, Zhao J, Li F, Chan A S C 
and Kwong F Y 2010 Highly Enantioselective and Efficient Organocatalytic Aldol Reaction of Acetone and $\beta, \gamma$-Unsaturated $\alpha$-Keto Ester Org. Lett. 12 5616; For review, see: (d) Melchiorre P 2012 Cinchona-based Primary Amine Catalysis in the Asymmetric Functionalization of Carbonyl Compounds Angew. Chem. Int. Ed. 51 9748

4. (a) Ricci A, Bernardi L, Gioia C, Vierucci S, Robitzer M and Quignard F 2010 Chem. Commun. 6288; (b) Shen C, Shen F, Xia H, Zhang P and Chen X 2011 Tetrahedron: Asymm. 22 708; (c) Agarwal J and Peddinti R K 2011 J. Org. Chem. 763502

5. (a) Bertelsen S, Halland N, Bachmann S, Marigo M, Braunton A and Jorgensen K A 2005 Organocatalytic asymmetric $\alpha$-bromination of aldehydes and ketones Chem. Commun. 4821; (b) Jensen K L, Franke P T, Nielsen L T, Daasbjerg K and Jørgensen K A 2010 Anodic Oxidation and Organocatalysis: Direct Regioand Stereoselective Access to meta-Substituted Anilines by $\alpha$-Arylation of Aldehydes Angew. Chem. Int. Ed. 49 129; (c) Alba A, Company X, Moyano A and Rios R 2009 Formal Highly Enantioselective Organocatalytic Addition of Alkyl Anions to $\alpha, \beta$-Unsaturated Aldehydes: Application to the Synthesis of IsotopeEnantiomers Chem. Eur. J. 15 11095; (d) Kano T, Mii H and Maruoka K 2009 Direct Asymmetric Benzoyloxylation of Aldehydes Catalyzed by 2-Tritylpyrrolidine $J$. Am. Chem. Soc. 131 3450; (e) Aggarwal V K, Lopin C and Sandrinelli F 2003 New insights in the mechanism of amine catalyzed epoxidation: Dual role of protonated ammonium salts as both phase transfer catalysts and activators of oxone J. Am. Chem. Soc. 1257596

6. (a) Hickmott P W 1982 Enamines: Recent advances in synthetic, spectroscopic, mechanistic, and stereochemical aspects-i Tetrahedron 38 1920; (b) Rappaport Z 1994 In Chemistry of Enamines (Chichester: Wiley); (c) Hine J and Evangelista R A 1980 Iminium-ion formation and deuterium exchange by acetone in the presence of pyrrolidine, pyrazolidine, Isoxazolidine, and their acyclic analogs J. Am. Chem. Soc. 1021649

7. (a) Witkop B 1956 Imine-Enamine Systems and the Mechanism of their Oxidation J. Am. Chem. Soc. 78 2873; (b) Clark R A and Parker D C 1971 Imineenamine tautomerism. I. 2-(N-Cyclohexylimino)-1,3diphenylpropane J. Am. Chem. Soc. 93 7257; (c) Boyd D R, Jennings W B and Waring L C 1986 Dynamic stereochemistry of imines and derivatives. 19. Mutarotation and E-Z isomerization of chiral imines in [2H4]methanol solution J. Org. Chem. 51992

8. Wurtz A 1872 On an aldehyde-alcohol Bull. Soc. Chim. Fr. 17436

9. (a) Smith M B and March J 2001 In Advanced Organic Chemistry 5th ed. (New York: Wiley Interscience) p.1218; (b) Wade L G 2005 Organic Chemistry 6th ed. (New Jersey: Prentice Hall) p.1056; (c) Mahrwald R 2004 In Modern Aldol Reactions Vol. 1 and 2 (Weinheim, Germany: Wiley-VCH Verlag) p.1218

10. (a) Li J J 2004 In Contemporary Drug Synthesis (New Jersey: Wiley-Interscience) 118; (b) Schetter B and Mahrwald R 2006 Modern Aldol Methods for the Total Synthesis of Polyketides Angew. Chem. Int. Ed. 45 7506; (c) Paterson I and Mansuri M M 1985 Recent developments in the total synthesis of macrolide antibiotic
Tetrahedron 41 3569; (d) Masamune S, Bates G S and Corcoran J W 1977 Electrocyclic Ring Opening Reactions of Ethylene Oxides Angew. Chem. Int. Ed. 16 585; For reviews on asymmetric aldol reaction, see: (e) Trost B M and Brindle C S 2010 The direct catalytic asymmetric aldol reaction Chem. Soc. Rev. 39 1600; (f) Geary L M and Hultin P G 2009 The State of the Art in asymmetric induction: The aldol reaction as a case study Tetrahedron: Asymm. 20 131; (g) Saito S and Yamamoto H 2004 Design of Acid-Base Catalysis for the Asymmetric Direct Aldol Reaction Acc. Chem. Res. 37 570; (h) Machajewski T D and Wong C-H 2000 The Catalytic Asymmetric Aldol Reaction Angew. Chem. Int. Ed. 39 1352

11. For review, see: (a) Mahrwald R 1999 Diastereoselection in Lewis-Acid-Mediated Aldol Additions Chem. Rev. 99 1095; (b) Johnson J S, Evans D A 2000 Chiral Bis(oxazoline) Copper(II) Complexes: Versatile Catalysts for Enantioselective Cycloaddition, Aldol, Michael, and Carbonyl Ene Reactions Acc. Chem. Res. 33 325; (c) Casiraghi G, Battistini L, Curti C, Rassu G and Zanardi F 2011 The Vinylogous Aldol and Related Addition Reactions: Ten Years of Progress Chem. Rev. 1113076

12. List B, Lerner R A and Barbas III C F 2000 ProlineCatalyzed Direct Asymmetric Aldol Reactions J. Am. Chem. Soc. 1222395

13. (a) Tong $\mathrm{S} \mathrm{T}$, Harris $\mathrm{P} \mathrm{W}$ R, Barker D and Brimble M A 2008 Use of (S)-5-(2-Methylpyrrolidin-2-yl)-1 Htetrazole as a Novel and Enantioselective Organocatalyst for the Aldol Reaction Eur. J. Org. Chem. 164; (b) Zheng J F, Li Y X, Zhang S Q, Yang S T, Wang X M, Wang Y Z, Bai J and Liu F A 2006 New N-terminal prolyl-dipeptide derivatives as organocatalysts for direct asymmetric aldol reaction Tetrahedron Lett. 47 7793; (c) Moorthy J N and Saha S 2009 Highly Diastereo- and Enantioselective Aldol Reactions in Common Organic Solvents Using $N$-Arylprolinamides as Organocatalysts with Enhanced Acidity Eur. J. Org. Chem. 739; (d) Pedrosa R, Andrés J M, Manzano R and Rodríguez P 2010 L-Prolinamides Derived from Chiral and Achiral 1,2-Diamines as Useful Bifunctional Organocatalysts for Direct Diastereo- and Enantioselective Aldol Reaction Eur. J. Org. Chem. 5310; (e) Gruttadauria M, Giacalone F, Marculescu A M, Meo P L, Riela S and Noto R 2007 Hydrophobically Directed Aldol Reactions: Polystyrene-Supported L-Proline as a Recyclable Catalyst for Direct Asymmetric Aldol Reactions in the Presence of Water Eur. J. Org. Chem. 4688; (f) J Huang, Zhang X and Armstrong D W 2007 Highly Efficient Asymmetric Direct Stoichiometric Aldol Reactions on/in Water Angew. Chem. Int. Ed. 469073 and the references cited therein; (g) Melchiorre P, Marigo M, Carlone A and Bartoli G 2008 Angew. Chem. Int. Ed. 47 6138; (h) Dondoni A and Massi A 2008 Asymmetric Organocatalysis: From Infancy to Adolescence Angew. Chem. Int. Ed. 474638

14. (a) Agarwal J and Peddinti R K 2010 Highly efficient and solvent-free direct aldol reaction catalyzed by glucosamine-derived prolinamide Tetrahedron: Asymm. 21 1906; (b) Guillena G, Hita M C and Nájera C 
$2007 \alpha$-Chloroacetone as a donor in the BINAM-1prolinamide organocatalyzed aldol reaction: Application to the enantioselective synthesis of $\alpha, \beta$-epoxy ketones Tetrahedron: Asymm. 18 1272; (c) Viozquez S F, Banon-Caballero A, Guillena G, Najera C and GomezBengoa E 2012 Enantioselective direct aldol reaction of $\alpha$-keto esters catalyzed by $\left(\mathrm{S}_{\mathrm{a}}\right)$-binam-D-prolinamide under quasi solvent-free conditions Org. Biomol. Chem. 104029

15. Nie J, Li X-J, Zheng D-H, Zhang F-G, Cui S and Ma J-A 2011 Chiral bifunctional thiourea-catalyzed enantioselective aldol reaction of trifluoroacetaldehyde hemiacetal with aromatic ketones J. Fluorine Chem. 132 468

16. Vincent J-M, Margottin C, Berlande M, Cavagnat D, Buffeteau T and Landais Y 2007 A concise organocatalytic and enantioselective synthesis of isotetronic acids Chem. Commun. 4782

17. Chen J R, Lu H H, Li X Y, Cheng L, Wan J and Xiao W 2005 Readily Tunable and Bifunctional L-Prolinamide Derivatives: Design and Application in the Direct Enantioselective Aldol Reactions Org. Lett. 7 4543

18. Hernández J G and Juaristi E 2011 Asymmetric Aldol Reaction Organocatalyzed by (S)-Proline-Containing Dipeptides: Improved Stereoinduction under SolventFree Conditions J. Org. Chem 761464
19. Tang $\mathrm{H}$ and Carter R G 2008 N-(p-Dodecylphenylsulfonyl)-2-pyrrolidinecarboxamide: A Practical Proline Mimetic for Facilitating Enantioselective Aldol Reactions Org. Lett. 104649

20. Inoue H, Kikuchi M, Ito J and Nishiyama H 2008 Chiral Phebox-rhodium complexes as catalysts for asymmetric direct aldol reaction Tetrahedron 64493

21. Giacalone F, Gruttadauria M, Meo P L, Riela S and Noto R 2008 New Simple Hydrophobic Proline Derivatives as Highly Active and Stereoselective Catalysts for the Direct Asymmetric Aldol Reaction in Aqueous Medium Adv. Synth. Catal. $\mathbf{3 5 0} 2747$

22. Vanlaldinpuia K and Bez G 2011 Useful methods for the synthesis of isopropylidenes and their chemoselective cleavage Tetrahedron Lett. 523759

23. Guo J and Frost J W 2002 Biosynthesis of 1-Deoxy-1imino-D-erythrose 4-Phosphate:A Defining Metabolite in the Aminoshikimate Pathway J. Am. Chem. Soc. 124 528

24. Vanladinpuia K, Sema H A, Rokum L and Bez G 2010 An excellent method for Cbz protection of amines Chem. Lett. 228

25. Rulli G, Duangdee N, Baer K, Hummel W, Berkessel A and Groger H 2011 Direction of Kinetically versus Thermodynamically Controlled Organocatalysis and Its Application in Chemoenzymatic Synthesis Angew. Chem. Int. Ed. $\mathbf{5 0} 7944$ 